\title{
A CONCEPTUAL APPROACH TO THE PROBLEM OF ACTION-ANGLE VARIABLES
}

\author{
NGUYEN TIEN ZUNG
}

\begin{abstract}
In this paper we develop a general conceptual approach to the problem of existence of action-angle variables for dynamical systems, which establishes and uses the fundamental conservation property of associated torus actions: anything which is preserved by the system is also preserved by the associated torus actions. This approach allows us to obtain, among other things: a) the shortest and most easy to understand conceptual proof of the classical Arnold-Liouville-Mineur theorem; b) basically all known results in the literature about the existence of action-angle variables in various contexts can be recovered in a unifying way, with simple proofs, using our approach; c) new results on action-angle variables in many different contexts, including systems on contact manifolds, systems on presymplectic and Dirac manifolds, action-angle variables near singularities, stochastic systems, and so on. Even when there are no natural action variables, our approach still leads to useful normal forms for dynamical systems, which are not necessarily integrable.
\end{abstract}

\section{Contents}

1. Introduction

2. Fundamental conservation property of Liouville torus actions

3. Action-angle variables on manifolds with a differential 2-form 10

4. Action-angle variables on contact manifolds 15

5. Action-angle variables on Dirac and Poisson manifolds 20

6. Torus actions and action-angle variables near singularities 34

7. Some final remarks 39

Acknowledgement 40

Compliance with ethical standards 40

References 41

\section{INTRODUCTION}

Action-angle variables play a fundamental role in classical and quantum mechanics. They are the starting point of the famous Kolmogorov-Arnold-Moser theory about the

Date: This version: February 2018, accepted for publication in ARMA.

1991 Mathematics Subject Classification. 37G05, 37J35,70H06,70H45.

Key words and phrases. torus actions, action-angle variables, integrable system, contact, symplectic, Poisson structure, Dirac manifold, presymplectic. 
persistence of quasi-preridicity of the motion of integrable Hamiltonian systems under perturbations (see, e.g., [11, 19, 36]). They are also the starting point of geometric quantization rules which go back to the works of Bohr, Sommefeld, Epstein and Einstein in early 20th century (see, e.g., [6]), and also of semi-classical quantization of integrable Hamiltonian systems (see, e.g., 64). The action functions are already written by contour integral formulas (see Formula (5.17)) in the works of Burgers [12, Einstein [24], Levi-Civita [42] and other physicists, and they also play the role of adiabatic invariants in mechanics and physics. The quasi-periodicity of the movement of general proper integrable Hamiltonian systems in angle variables was discovered by Liouville in mid 19th century [46]. The first essentially complete proof of the theorem about the existence of action-angle variables near a Liouville torus on a symplectic manifold, which is often called Arnold-Liouville theorem, is due to the astrophysicist Mineur [48, 49], who was also motivated by the quantization problem. There are now many books which contain a proof of this theorem, see for example [3, 28, 29, 45, 47]. An interesting account on the early history of action-angle variables can be found in a recent paper by Féjoz [26]. However, it seems to us that the available proofs in the literature are not yet "very natural": they contain arguments which are a bit tricky, and do not generalize easily to other contexts without a lot of additional work.

There have been generalizations of Arnold-Liouville-Mineur action-angle variables theorem to various contexts, including noncommutatively integrable systems (see, e.g., [54, [53, 17]), systems on almost-symplectic manifolds [25], on contact manifolds (see, e.g., [37, 32]), on Poisson manifolds [40], and so on. Action-angle variables near singularities of integrable systems have also been studied (see, e.g., [20, 52, 64, 67, 72, 73]). However, there are many other natural contexts, including presymplectic and Dirac manifolds, for which there was no systematic study of the existence of action-angle variables, as far as I know.

In this paper, we develop a new general conceptual approach for the study of existence of action-angle variables, which, in our opinion, is the most natural, easy to understand, and can be applied to a multitude of very different situations. If one is interested only in the classical Arnold-Liouville-Mineur theorem, then this approach will provide a very short and simple self-contained proof, only a few pages long. (Our proof in this paper finishes at page 13, but that's because we have a long introduction and prove many other general results of independent interest along the way). Using our approach, one can also recover practically all known results in the literature about the existence of action-angle variable in various contexts, in a unifying way, with simple proofs, and obtain a series of new results.

Our approach is based on the "toric philosophy" and consists of 3 parts:

i) Existence of associated torus actions for dynamical systems. For example, for integrable systems near Liouville tori, these actions are nothing but the Liouville torus actions, which are provided by the classical Liouville theorem dating back to mid 19th century [46]. For general analytic vector fields near singular points, these associated torus actions are exactly the ones appearing in the (formal or analytic) PoincaréBirkhoff normalization [69, 72]. 
ii) The fundamental conservation property of these associated torus actions. This is a fundamental property of dynamical systems, but whose proof is not very difficult and is provided in this paper. The philosophical idea behind this property comes from the notion of double commutant in algebra.

iii) Simultaneous normalization of the associated torus action together with the underlying geometric structure preserved by it leads to action-angle variables (if case of (quasi-)Hamiltonian systems) or other interesting normal forms.

The organization of this paper is as follows:

In Section 2 we recall some basic notions about integrable dynamical systems (which can be non-Hamiltonian), Liouville torus actions near Liouville tori, and establish the fundamental conservation property for Liouville torus actions. (Theorems 2.2, 2.4, 2.5 and 2.6 ).

In Section 3 we show the existence of action-angle variables for integrable Hamiltonian systems on manifolds with a differential 2-form, which is not necessarily closed nor nondegenerate. The classical Arnold-Liouville-Mineur theorem, as well as results obtained by Nekhoroshev [54] and Fassò-Sansonetto [25] are presented as special cases of our general results. We pay particular attention to (over-determined) action-angle variables for integrable Hamiltonian systems on presymplectic manifolds, which can happen quite often in practice (for example, by looking at the isoenergy submanifolds of an integrable Hamiltonian system on a symplectic manifold), and which leads to an interesting generalization of (integral) affine geometry, which we call (integral) co-affine geometry. Unlike affine manifolds, co-affine manifolds have a lot of local invariants (e.g., curvature). A special case of toric integrable Hamiltonian systems on presymplectic manifolds whose base spaces are flat co-affine manifolds was studied recently by Tudor Ratiu and the author [56].

In Section 4 we study the problem of action-angle variables of contact manifolds, which is one of the problems posed by V.I. Arnold in 1995 [2]. Our result about action-angle variables in the generic (transversal) case is a significant improvement of earlier results by Banyaga-Molino [5] and Jovanovic [32]. We also obtain action-angle variables for the non-transversal case, which has not been studed by other authors, as far as we know.

Section 5 is the longest section of this paper, and is devoted to a study of integrable Hamiltonian systems on Dirac manifolds and their action-angle variables. There are two reasons why we are so interested in general Dirac manifolds: i) There are many dynamical systems which cannot be written as Hamiltonian systems on symplectic or Poisson manifolds but which can be written as Hamiltonian systems on Dirac manifolds; ii) The problem of action-angle variables on Dirac manifolds had not been treated by any other author, as far as we know. In the special case, when the Dirac structure turns out to be a Poisson structure, we recover the main results of Laurent-MirandaVanhaecke [40], with a simpler proof.

In Section 6 we discuss the problem of action-angle variables near singularities of dynamical systems and its relations with local normalizations à la Poincaré-Birkhoff and associated torus actions [67, 52, 69, 70, 72]. 
Finally, in Section 7 we indicate how our general conceptual approach can lead to action-angle variables or at least interesting normal forms in other contexts: infinitedimensional integrable systems, stochastic systems, quantum and semi-classical systems, etc.

\section{Fundamental CONSERVATION PROPERTy OF LiOUVille TORUS ACTIONS}

2.1. Integrable systems and Liouville tori. Let us recall the following natural notion of integrability of dynamical systems which are not necessarily Hamiltonian (see, e.g., [8, 69, 73]):

A $m$-tuple $\left(X_{1} \ldots, X_{p}, F_{1}, \ldots, F_{q}\right)$, where $p \geq 1, q \geq 0, p+q=m, X_{i}$ are vector fields on a $m$-dimensional manifold $M$ and $F_{j}$ are functions on $M$, is called an integrable system of type $(p, q)$ on $M$ if it satisfies the following commutativity and non-triviality conditions:

i) $\left[X_{i}, X_{j}\right]=0 \quad \forall i, j=1, \ldots, p$,

ii) $X_{i}\left(F_{j}\right)=0 \quad \forall i \leq p, j \leq q$,

iii) $X_{1} \wedge \ldots \wedge X_{p} \neq 0$ and $d F_{1} \wedge \ldots \wedge d F_{q} \neq 0$ almost everywhere on $M$.

A dynamical system given by a vector field $X$ on a manifold $M$ is called integrable if there is an integrable system $\left(X_{1} \ldots, X_{p}, F_{1}, \ldots, F_{q}\right)$ of some type $(p, q)$ on $M$ with $X_{1}=X$.

The above integrability notion is also called non-Hamiltonian integrability. It does not mean that the system cannot admit any Hamiltonian structure, it simply means that we forget about the Hamiltonian structure, and look only at commuting flows and first integrals in the definition. If a Hamiltonian system on a symplectic manifold is integrable in the sense of Liouville or in noncommutative sense then it is also integrable in the above sense. But systems with non-holonomic constraints, which are a priori non-Hamiltonian, can also be integrable in the above sense.

By a level set of an integrable system $\left(X_{1} \ldots, X_{p}, F_{1}, \ldots, F_{q}\right)$ we mean a connected component $N$ of a joint level set

$$
\left\{F_{1}=\text { const }, \ldots, F_{q}=\text { const }\right\} \text {. }
$$

Notice that, by definition, the vector fields $X_{1}, \ldots, X_{p}$ are tangent to the level sets of the system. We will say that the system $\left(X_{1} \ldots, X_{p}, F_{1}, \ldots, F_{q}\right)$ is regular at $N$ if $X_{1} \wedge \ldots \wedge X_{p} \neq 0$ and $d F_{1} \wedge \ldots \wedge d F_{q} \neq 0$ everywhere on $N$. We will say that the system is proper if the map $\left(F_{1}, \ldots, F_{q}\right): M \rightarrow \mathbb{R}^{q}$ is a proper topological map (so that each level set is compact) and the system is regular at almost every level set.

The following theorem about the existence of a system-preserving torus action near a compact regular level set of an integrable system is essentially due to Liouville [46]:

Theorem 2.1 (Liouville's theorem). Assume that $\left(X_{1}, \ldots, X_{p}, F_{1}, \ldots, F_{q}\right)$ in an integrable system of type $(p, q)$ on a manifold $M$ which is regular at a compact level set $N$. Then in a tubular neighborhood $\mathcal{U}(N)$ there is, up to automorphisms of $\mathbb{T}^{p}$, a unique free torus action

$$
\rho: \mathbb{T}^{p} \times \mathcal{U}(N) \rightarrow \mathcal{U}(N)
$$


which preserves the system (i.e. the action preserves each $X_{i}$ and each $F_{j}$ ) and whose orbits are regular level sets of the system. In particular, $N$ is diffeomorphic to $\mathbb{T}^{p}$, and

$$
\mathcal{U}(N) \cong \mathbb{T}^{p} \times B^{q}
$$

with periodic coordinates $\theta_{1}(\bmod 1), \ldots, \theta_{p}(\bmod 1)$ on the torus $\mathbb{T}^{p}$ and coordinates $\left(z_{1}, \ldots, z_{q}\right)$ on a q-dimensional ball $B^{q}$, such that $F_{1}, \ldots, F_{q}$ depend only on the variables $z_{1}, \ldots, z_{q}$, and the vector fields $X_{i}$ are of the type

$$
X_{i}=\sum_{j=1}^{p} a_{i j}\left(z_{1}, \ldots, z_{q}\right) \frac{\partial}{\partial \theta_{j}} .
$$

A system of coordinates

$$
\left(\theta_{1}(\bmod 1), \ldots, \theta_{p} \quad(\bmod 1), z_{1}, \ldots, z_{q}\right)
$$

on $\mathcal{U}(N) \cong \mathbb{T}^{p} \times B^{q}$ given by the above theorem will be called a Liouville system of coordinates.

The proof of the above theorem is absolutely similar to the case of integrable Hamiltonian systems on symplectic manifolds, see, e.g., [8, 73]. It consists of the following main points:

1) The map $\left(F_{1}, \ldots, F_{q}\right): \mathcal{U}(N) \rightarrow \mathbb{R}^{q}$ from a tubular neighborhood of $N$ to $\mathbb{R}^{q}$ is a topologically trivial fibration by the level sets, due to the compactness of $N$ and the regularity of $\left(F_{1}, \ldots, F_{q}\right)$ (attention: if $\left(F_{1}, \ldots, F_{q}\right)$ is not regular at $N$ then this fibration may be non-trivial and may be twisted even if the level sets are smooth);

2) The vector fields $X_{1}, \ldots, X_{p}$ generate a transitive action of $\mathbb{R}^{p}$ on the level sets near $N$, and the level sets are compact and of dimension $p$, which imply that each level set $N_{f_{1}, \ldots, f_{q}}$ is a $p$-dimensional compact quotient of $\mathbb{R}^{p}$ by a discrete group $\Gamma$, i.e. a torus.

3) Consider a local section $S$ to the foliation by the level sets, i.e. a small disk which intersects each level set $N_{f_{1}, \ldots, f_{q}}$ near $N$ transversally at one point denoted by $s_{f_{1}, \ldots, f_{q}}$. Let $\varphi_{X_{i}}^{t}$ be the flow of $X_{i}$. Composing the map $\left(t_{1}, \ldots, t_{p}\right) \mapsto \varphi_{X_{1}}^{t_{1}} \circ \ldots \circ \varphi_{X_{p}}^{t_{p}}\left(s_{f_{1}, \ldots, f_{q}}\right)$ from $\mathbb{R}^{q} / \Gamma$ to $N_{f_{1}, \ldots, f_{q}}$ with an isomorphism from $\mathbb{R}^{q} / \mathbb{Z}^{q}$ to $\mathbb{R}^{q} / \Gamma$, one gets angular coordinates $\theta_{1}, \ldots, \theta_{p}$ which turn the $X_{i}$ into constant vector fields on each level set $N_{f_{1}, \ldots, f_{q}}$.

Theorem 2.1 shows that the flow of the vector field $X=X_{1}$ of an integrable system is quasi-periodic under some natural compactness and regularity conditions. This is the most fundamental geometrical property of proper integrable dynamical systems.

Due to the above theorem, each $p$-dimensional compact level set $N$ of an integrable system of type $(p, q)$ on which the system is regular is called a Liouville torus, and the torus $\mathbb{T}^{p}$-action in a tubular neighborhood $\mathcal{U}(N)$ of $N$ which preserves the system is called the Liouville torus action. Notice that this action is uniquely determined by the system, up to an automorphism of $\mathbb{T}^{p}$.

2.2. Fundamental conservation property of Liouville torus actions. The basic idea is the following meta-theorem, which is a new kind of conservation laws: 


\section{Everything which is preserved by a dynamical system is also preserved by its associated torus actions.}

In other words, the associated torus actions are double commutants for dynamical systems. Some instances of this meta-theorem can be found, e.g., in [69, 172, 73].

In this subsection, we will turn the above meta-theorem into some rigorous theorems about the fundamental conservation property of Liouville torus actions, which play the role of associated torus actions for integrable systems near Liouville tori.

Remark that the idea of double commutant torus actions having the fundamental conservation property is more general and also works for singularities of dynamical systems which are not necessarily integrable, and also for stochastic and quantum systems, see Section 6 and Section 7 of this paper.

Theorem 2.2 (Fundamental conservation property, 1). Let $N$ be a Liouville torus of an integrable system $\left(X_{1} \ldots, X_{p}, F_{1}, \ldots, F_{q}\right)$ on a manifold $M$, and $\mathcal{G} \in \Gamma\left(\otimes^{h} T M \otimes^{k} T^{*} M\right)$ be a tensor field on $M$ which is preserved by all the vector fields of the system: $\mathcal{L}_{X_{i}} \mathcal{G}=0$ $\forall i=1, \ldots, p$. Then the Liouville torus $\mathbb{T}^{p}$-action on a tubular neighborhood $\mathcal{U}(N)$ of $N$ in $M$ also preserves $\mathcal{G}$.

Remark 2.3. The above theorem first appeared in my unpublished preprint in 2012 (arXiv:1204.3865), and was also included in the lecture notes of a course that I gave on integrable non-Hamiltonian systems in CRM Barcelona in 2013 (see [9]), but it has not been published in any peer-reviewed research journal before. The following proof of the theorem borrows ideas from the theory of spectral sequences in algebraic topology.

Proof. Fix a Liouville coordinate system

$$
\left(\theta_{1} \quad(\bmod 1), \ldots, \theta_{p} \quad(\bmod 1), z_{1}, \ldots, z_{q}\right)
$$

in a tubular neighborhood $\mathcal{U}(N)$ of $N$ as given by Theorem 2.1. The Liouville torus action is generated by the vector fields $\frac{\partial}{\partial \theta_{i}}$.

We will make a filtration of the space $\Gamma\left(\otimes^{h} T M \otimes^{k} T^{*} M\right)$ of tensor fields of covariant order $k$ and contravariant order $h$ as follows:

The subspace $T_{s}^{h, k}$ consists of sections of $\otimes^{h} T M \otimes^{h} T^{*} M$ whose expression in Liouville coordinates contains only terms which are, up to a permutation of the factors, of the type

$$
\frac{\partial}{\partial \theta_{i_{1}}} \otimes \ldots \otimes \frac{\partial}{\partial \theta_{i_{a}}} \otimes \frac{\partial}{\partial z_{j_{1}}} \otimes \ldots \otimes \frac{\partial}{\partial z_{j_{b}}} \otimes d \theta_{i_{1}^{\prime}} \otimes \ldots \otimes d \theta_{i_{c}^{\prime}} \otimes d z_{j_{1}^{\prime}} \otimes \ldots \otimes d z_{j_{d}^{\prime}}
$$

with $b+c \leq s$. For example,

$$
T_{0}^{h, k}=\left\{\sum_{i, j^{\prime}} f_{i, j^{\prime}} \frac{\partial}{\partial \theta_{i_{1}}} \otimes \ldots \otimes \frac{\partial}{\partial \theta_{i_{h}}} \otimes d z_{j_{1}^{\prime}} \otimes \ldots \otimes d z_{j_{k}^{\prime}}\right\} .
$$

Put $T_{-1}^{h, k}=\{0\}$. It is clear that

$$
\{0\}=T_{-1}^{h, k} \subset T_{0}^{h, k} \subset T_{1}^{h, k} \subset \ldots \subset T_{h+k}^{h, k}=\Gamma\left(\otimes^{k} T M \otimes^{h} T^{*} M\right) .
$$


Observe that the vector fields $\frac{\partial}{\partial \theta_{i}}$ and the differential forms $d z_{j}$ are invariant under the flow of any of the vector fields $X_{\alpha}(\alpha=1, \ldots, p)$, while the Lie derivative $\mathcal{L}_{X_{\alpha}} \frac{\partial}{\partial z_{j}}$ (respectively, $\mathcal{L}_{X_{i}} d \theta_{i}$ ) is a combination of vector fields $\frac{\partial}{\partial \theta_{1}}, \ldots, \frac{\partial}{\partial \theta_{p}}$ (respectively, of 1-forms $\left.d z_{1}, \ldots, d z_{q}\right)$. It follows immediately from this observation that the above filtration is stable under the Lie derivative of the vector fields $X_{1}, \ldots, X_{p}$, i.e., we have

$$
\mathcal{L}_{X_{\alpha}} \Lambda \in T_{s}^{h, k} \forall s=0, \ldots, k+h, \forall \Lambda \in T_{s}^{h, k}, \forall \alpha=1, \ldots, p .
$$

Since $\mathcal{L}_{X_{\alpha}} \mathcal{G}=0$ for all $\alpha=1, \ldots, p$ by our hypothesis, and the Liouville torus action commutes with the vector fields $X_{\alpha}$, we also have that $\mathcal{L}_{X_{\alpha}} \overline{\mathcal{G}}=0$, where the overline means the average of a tensor with respect to the Liouville torus action. Thus we also have

$$
\mathcal{L}_{X_{\alpha}} \hat{\mathcal{G}}=0 \forall \alpha=1, \ldots, p,
$$

where

$$
\hat{\mathcal{G}}=\mathcal{G}-\overline{\mathcal{G}}
$$

has average equal to 0 .

We will show by induction that $\hat{\mathcal{G}} \in T_{s}^{h, k}$ for every $s$ going down from $h+k$ to -1 . Of course we have $\hat{\mathcal{G}} \in T_{h+k}^{h, k}=\Gamma\left(\otimes^{k} T M \otimes^{h} T^{*} M\right)$, and at the end of the induction process we will get $\hat{\mathcal{G}} \in T_{-1}^{h, k}=\{0\}$, i.e. $\hat{\mathcal{G}}=0$.

Assume that we already have $\hat{\mathcal{G}} \in T_{s}^{h, k}$ for some $s$ with $h+k \geq s \geq 0$. Let us show that $\hat{\mathcal{G}} \in T_{s-1}^{h, k}$.

Consider a possible monomial term $\Psi$ in the expression of $\hat{\mathcal{G}}$ which belongs to $T_{s}^{h, k}$ but does not belong to $T_{s-1}^{h, k}$. Up to a permutation of the factors, $\Psi$ is of the type

$$
\Psi=\psi \frac{\partial}{\partial \theta_{i_{1}}} \otimes \ldots \otimes \frac{\partial}{\partial \theta_{i_{a}}} \otimes \frac{\partial}{\partial z_{j_{1}}} \otimes \ldots \otimes \frac{\partial}{\partial z_{j_{b}}} \otimes d \theta_{i_{1}^{\prime}} \otimes \ldots \otimes d \theta_{i_{c}^{\prime}} \otimes d z_{j_{1}^{\prime}} \otimes \ldots \otimes d z_{j_{d}^{\prime}}
$$

such that $b+c=s$, with some coefficient function $\psi$.

According to the observation that we made above, in the decomposition of the Lie derivative $\mathcal{L}_{X_{\alpha}} \Psi$ by the Leibniz rule, all the terms belong to $T_{s-1}^{h, k}$ except maybe the term

$$
X_{\alpha}(\psi) \frac{\partial}{\partial \theta_{i_{1}}} \otimes \ldots \otimes \frac{\partial}{\partial \theta_{i_{a}}} \otimes \frac{\partial}{\partial z_{j_{1}}} \otimes \ldots \otimes \frac{\partial}{\partial z_{j_{b}}} \otimes d \theta_{i_{1}^{\prime}} \otimes \ldots \otimes d \theta_{i_{c}^{\prime}} \otimes d z_{j_{1}^{\prime}} \otimes \ldots \otimes d z_{j_{d}^{\prime}}
$$

which is a monomial term belonging to $T_{s}^{h, k} \backslash T_{s-1}^{h, k}$ if $X_{\alpha}(\psi) \neq 0$. But $\mathcal{L}_{X_{\alpha}} \hat{\mathcal{G}}=0$, which implies in particular that $\mathcal{L}_{X_{\alpha}} \hat{\mathcal{G}} \in T_{s-1}^{h, k}$ and it cannot contain any monomial term in $T_{s}^{h, k} \backslash T_{s-1}^{h, k}$, and so we must have $X_{\alpha}(\psi)=0$ (for every $\alpha=1, \ldots, p$ ), i.e. $\psi$ is invariant with respect to the vector field $X_{1}, \ldots, X_{p}$. It means that $\psi$ is constant on each Liouville torus. On the other hand, by the definition of $\hat{G}$, the mean value of $\psi$ on each Liouville torus is zero, so in fact $\psi$ is identically zero, and there is no monomial term of $\mathcal{G}$ in $T_{s}^{h, k} \backslash T_{s-1}^{h, k}$, i.e. we have $\mathcal{G} \in T_{s-1}^{h, k}$. 
Thus, we have shown by induction that $\hat{\mathcal{G}}=0$, i.e. $\mathcal{G}=\overline{\mathcal{G}}$ is invariant with respect to the Liouville torus action.

Theorem 2.2 admits the following slightly stronger version:

Theorem 2.4 (Fundamental conservation property, 2). Let $N$ be a Liouville torus of an integrable system $\left(X_{1} \ldots, X_{p}, F_{1}, \ldots, F_{q}\right)$ on a manifold $M$, such that in a tubular neighborhood $\mathcal{U}(N) \cong \mathbb{T}^{p} \times B^{q}$ of $N$ the set of points $\mathbf{z} \in B^{q}$ such that the orbits of the vector field $X_{1}$ on the Liouville torus $\mathbb{T}^{p} \times\{\mathbf{z}\}$ are dense in the torus (i.e., the flow of $X_{1}$ is totally irrational on the torus) is a dense subset of $B^{q}$. Then any tensor field $\mathcal{G} \in \Gamma\left(\otimes^{h} T M \otimes^{k} T^{*} M\right)$ which is invariant with respect to $X_{1}$ is also invariant with respect to the Liouville torus $\mathbb{T}^{p}$-action on $\mathcal{U}(N)$.

Notice that in Theorem 2.4, we do not require $\mathcal{G}$ to be invariant with respect to $X_{2}, \ldots, X_{p}$. We only require it to be invariant with respect to the original vector field $X=X_{1}$ of an integrable dynamical system, and this invariance is often automatically obtained in practice, e.g., a Hamiltonian vector field will automatically preserve the symplectic structure, but the additional vector fields are not required to be symplectic.

The proof of Theorem 2.4 is absolutely similar to the proof of Theorem 2.2 and is based on the observation that, under the above assumptions about $X_{1}$, any function which is invariant with respect to $X_{1}$ is also invariant with respect to the Liouville torus action in $\mathcal{U}(N)$.

We will call the condition about dense orbits in Liouville tori imposed on the vector field $X_{1}$ in Theorem 2.4 the complete irrationality condition. Theorem 2.4 is stronger than Theorem 2.2, because Theorem 2.2 can be deduced from it by changing $X_{1}$ to a new vector field $X_{1}^{\prime}=\sum c_{i} X_{i}$, which is a linear combination of $X_{1}, \ldots, X_{p}$ with constant coefficients, and which satisfies the complete irrationality condition. For integrable Hamiltonian systems in the sense of Liouville, this complete irrationality is implied by the Kolmogorov's nondegeneracy condition in K.A.M theory, see, e.g., [19, 68].

The above theorem can be applied to many kinds of underlying geometric structures which are preserved by the systems, e.g. volume form (isochore systems), Riemannian metric, Nambu structure, symplectic or Poisson structure (Hamiltonian systems), symmetry groups or algebras (generated by vector fields, which are considered as tensors), and so on. However, there are some geometric structures, e.g., contact distributions and Dirac structures, which can't be written as tensors. To deal with them, we have to extend Theorem 2.2 to the case of subbundles of natural vector bundles preserved by the system, as will be explained below.

We will say that a tensor field $\mathcal{G}$ on $M$ is conformally conserved by the system $\left(X_{1} \ldots, X_{p}, F_{1}, \ldots, F_{q}\right)$ (or conformally invariant) if for each $i=1, \ldots, p$ there is a (smooth) function $f_{i}$ on $M$ such that $\mathcal{L}_{X_{i}} \mathcal{G}=f_{i} \cdot \mathcal{G}$.

Theorem 2.5 (Fundamental conservation property, 3). With the above notations, if a tensor field $\mathcal{G}$ is conformally conserved by the integrable system $\left(X_{1} \ldots, X_{p}, F_{1}, \ldots, F_{q}\right)$ in a neighborhood of a Liouville torus, or if $X_{1}$ satisfies the complete irrationality 
condition and $\mathcal{G}$ is conformally invariant with respect to $X_{1}$, then $\mathcal{G}$ is also conformally invariant with respect to the Liouville $\mathbb{T}^{p}$-action.

Proof. The proof of Theorem 2.5 can be reduced to the proof of Theorem 2.2. by multiplying $\mathcal{G}$ by an appropriate function.

Indeed, consider the case when $X_{1}$ satisfies the complete irrationality condition, and let $\mathcal{G} \in \Gamma\left(\otimes^{h} T M \otimes^{k} T^{*} M\right)$ be conformally invariant with respect to $X_{1}: \mathcal{L}_{X_{1}} \mathcal{G}=f \mathcal{G}$ for some function $f$. (The case when $\mathcal{G}$ is invariant with respect to $X_{1}, \ldots, X_{p}$ but wihout the irrationality condition can be reduced to this case by taking a linear combination $X_{1}^{\prime}=\sum_{i=1}^{p} c_{i} X_{i}$ with appropriate constant coefficients $c_{i}$ so that $X_{1}^{\prime}$ is completely irrational and preserves $\mathcal{G}$ ).

We assume that $\mathcal{G} \neq 0$, and want to show that there is a function $g$ such that $\exp (g) \mathcal{G}$ is invariant with respect to $X_{1}$. By conformal invariance, we have $\mathcal{L}_{X_{1}}(\exp (g) \mathcal{G})=$ $X_{1}(g) \exp (g) \mathcal{G}+f \exp (g) \mathcal{G}$, so the equation to solve is

$$
X_{1}(g)+f=0,
$$

which of course locally has a solution, which is unique up to a function which is constant on the orbits of $X_{1}$. The problem is that maybe it doesn't have a global smooth solution in $\mathcal{U}(N)$. So we have to show that a global solution in fact exists.

With respect to the filtration given in the proof of Theorem 2.2 , there is a number $s$ such that $\mathcal{G} \in T_{s}^{h, k} \backslash T_{s-1}^{h, k}$. Let

$$
h \frac{\partial}{\partial \theta_{i_{1}}} \otimes \ldots \otimes \frac{\partial}{\partial \theta_{i_{a}}} \otimes \frac{\partial}{\partial z_{j_{1}}} \otimes \ldots \otimes \frac{\partial}{\partial z_{i_{b}}} \otimes d \theta_{i_{1}^{\prime}} \otimes \ldots \otimes d \theta_{i_{c}^{\prime}} \otimes d z_{j_{1}^{\prime}} \otimes \ldots \otimes d z_{j_{d}^{\prime}}
$$

with $b+c=s$, up to a permutation of the factors, be a term of $\mathcal{G}$ of highest filtration degree $b+c=s$ with non-zero coefficient function $h$. Then the term of the same type in $\mathcal{L}_{X_{1}} \mathcal{G}$ has coefficient $X_{1}(h)$. Since $\mathcal{L}_{X_{1}} \mathcal{G}=f \mathcal{G}$ and $h \neq 0$ we must have $f=X_{1}(h) / h$. It implies (also due to the fact that $X_{1}$ is completely irrational) in particular that $h \neq 0$ everywhere in a small tubular neighborhood $\mathcal{U}(N)$ of $N$, and that the solutions in $\mathcal{U}(N)$ of the equation $X_{1}(g)+f=0$ are $g=-\ln (|h|)+$ const., and we are done.

Given a manifold $M$, the vector bundles on $M$ which can be obtained from the tangent and cotangent bundles $T M, T^{*} M$ and the trivial bundle $\mathbb{R} \times M$ by operations of taking sums and tensor products will be called natural vector bundles over $M$.

Theorem 2.6 (Fundamental conservation property, 4). Let $\mathcal{V}$ be a subbundle of a natural vector bundle over a manifold $M$, which is conserved by an integrable system on $M$, or by the completely irrational vector field $X_{1}$ of the system. Then the Liouville torus action near any Liouville torus of the system preserves $\mathcal{V}$.

Proof. Theorem 2.6 can be reduced to Theorem 2.5 by using a tensor field which is a volume element on each fiber of the subbundle $\mathcal{V}$. (The exterior product of the components of a basis of a vector space is a contravariant volume element of that vector space). This tensor field is not invariant with respect to the system in general, but it will be conformally invariant, and it characterizes the subbundle $\mathcal{V}$.

There is a small technical problem: maybe this volume tensor field cannot be defined globally due to the possible non-orientability of the vector subbundle $\mathcal{V}$ (i.e., the 
holonomy does not preserve the orientation of the fibers). But this situation can be remedied easily by taking a double covering of the system.

Remark 2.7. Any tensor field can be viewed as a linear map from a natural vector bundle to another natural vector bundle (e.g., a differential $k$-form on $M$ can be viewed as an anti-symmetric linear map from the bundle $\otimes^{k} T M$ to the trivial bundle $\mathbb{R}$ on $M$, or also a linear map from $\otimes^{k-1} T M$ to $T^{*} M$, etc.), and the graph of such a linear map (which also characterizes our tensor field) is a subbundle of the sum of the two bundles, and this sum is also a natural bundle. So any tensor field may be viewed as a subbundle of a natural vector bundle. For example, both differential 2-forms and 2 -vector fields can be viewed as 2-dimensional subbundles of $T M \oplus T^{*} M$. But the converse is not true.

Theorem 2.6 can be applied to invariant distributions, e.g. nonholonomic constraints and contact structures, to systems on Dirac manifolds (which is one of the origins of this paper), and so on.

Another generalization of the fundamental conservation property is to differential operators which are preserved by the system. In fact, we have the following result, which was proved recently by N.T. Thien and the author in [75] using the same method of filtration, and which is useful for the study of reduction and action-angle variables for stochastic and quantum systems:

Theorem 2.8 ([75]). If $\Lambda$ is a linear differential operator on $M$ which is preserved by an integrable system $\left(X_{1}, \ldots, X_{p}, F_{1}, \ldots, F_{q}\right)$, or by a completely irrational component $X_{1}$ of the system, then $\Lambda$ is also invariant with respect to the Liouville torus action in the neighborhood of any Liouville torus of the system.

\section{ACtion-ANGle VARIABles on MANifolds With A DifFERENTIAL 2-FORM}

In this section, we will give a very simple proof of the Arnold-Liouville-Mineur theorem about the existence of action-angle variables near an invariant torus of a Hamiltonian system which is integrable in the classical sense of Liouville. In fact, we will obtain action-angle variables in a more general setting, for systems on manifolds with a differential 2-form which is not necessarily closed or nondenegerate, and the Arnold-Liouville-Mineur theorem is just a particular case of this more general result.

Consider a manifold $M$ together with an arbitrary given differential 2-form $\omega$, which is not necessarily closed nor nondegenerate. We will say that a vector field $X$ on $(M, \omega)$ is a Hamiltonian vector field of a function $H$ with respect to $\omega$ if it satisfies the following two equations:

$$
X\lrcorner \omega=-d H
$$

and

$$
\mathcal{L}_{X} \omega=0 .
$$

When $d \omega=0$ then Equality (3.2) can be omitted because it is a consequence of Equality (3.1). When $d \omega \neq 0$ then, under the assumption that Equality (3.1) holds, 
Equality (3.2) is equivalent to the fact that $X$ lies in the kernel of $d \omega$ :

$$
X\lrcorner d \omega=0 .
$$

Definition 3.1. We will say that an integrable system $\left(X_{1}, \ldots, X_{p}, F_{1}, \ldots, F_{q}\right)$ on a manifold $(M, \omega)$, where $\omega$ is a given differential 2-form on $M$, is an integrable Hamiltonian system of type $(p, q)$ if there are $p$ functions $H_{1}, \ldots, H_{p}$ on $(M, \omega)$ such that $X_{i}$ is a Hamiltonian vector field of $H_{i}$ with respect to $\omega$ for all $i=1, \ldots, p$.

It follows immediately from Definition 3.1 that if we have a proper integrable system $\left(X_{1}, \ldots, X_{p}, F_{1}, \ldots, F_{q}\right)$ which is Hamiltonian on $(M, \omega)$ with the aid of Hamiltonian functions $H_{1}, \ldots, H_{p}$, then these functions $H_{1}, \ldots, H_{p}$ are common first integrals of the system, i.e. $X_{i}\left(H_{j}\right)=0$ for all $i, j=1, \ldots, p$. Indeed, $X_{i}\left(H_{j}\right)=\omega\left(X_{i}, X_{j}\right)$ is invariant with respect to the vector fields of the system, hence this function is constant on each Liouville torus. Moreover, the mean value of $X_{i}\left(H_{j}\right)$ on each Liouville torus (with respect to the Liouville $\mathbb{T}^{p}$-action) is zero, because

$$
X_{i}\left(H_{j}\right)=\sum_{k} a_{j k}(z) \frac{\partial H_{j}}{\partial \theta_{k}}
$$

in a Liouville coordinate system, which implies that the integration of $X_{i}\left(H_{j}\right)$ over a Liouville torus with respect to the invariant volume form $d \theta_{1} \wedge \ldots \wedge d \theta_{p}$ is zero. Hence $X_{i}\left(H_{j}\right)$ is identically zero.

The equality $X_{i}\left(H_{j}\right)=\omega\left(X_{i}, X_{j}\right)=0$ also means that, for proper integrable Hamiltonian systems on $(M, \omega)$ (where the 2 -form $\omega$ may be non-closed and degenerate), the Liouville tori are isotropic with respect to $\omega$. As an immediate consequence, we get the following inequalities:

$$
p \leq \operatorname{dim} M-\frac{\operatorname{rank} \omega}{2}, \quad q \geq \frac{\operatorname{rank} \omega}{2}
$$

In particular, if $\omega$ is nondegenerate then $\operatorname{rank} \omega=\operatorname{dim} M$ and $p \leq \frac{\operatorname{dim} M}{2}$.

Proposition 3.2. If $\left(X_{1}, \ldots, X_{p}, F_{1}, \ldots, F_{q}\right)$ is a proper integrable Hamiltonian system on $(M, \omega)$, where $\omega$ is an arbitrary differential 2-form, then the Liouville $\mathbb{T}^{p}$-action in the neighborhood of any Liouville torus is a Hamiltonian action with respect to $\omega$, i.e. the components of this action are generated by Hamiltonian vector fields.

Proof. Let $Z_{1}, \ldots, Z_{p}$ be the generators of the Liouville $\mathbb{T}^{p}$-action near some Liouville torus $N$. Since $\left.X_{i}\right\lrcorner d \omega=0 \forall i$, we also have $\left.Z_{i}\right\lrcorner d \omega=0 \forall i$. Together with $\mathcal{L}_{Z_{i}} \omega=0$ (according to the fundamental conservation property), it implies that $\left.d\left(Z_{i}\right\lrcorner \omega\right)=0$, i.e., $\left.Z_{i}\right\lrcorner \omega$ is closed in a neighborhood $\mathcal{U}(N)$ of any Liouville torus $N$, hence it is exact (because its pull-back to a Liouville torus is zero), therefore there exists a $\mathbb{T}^{p}$-invariant function $\mu_{i}$ such that $\left.Z_{i}\right\lrcorner \omega=-d \mu_{i}$. Thus, the Liouville $\mathbb{T}^{p}$-action is Hamiltonian with momentum map $\left(\mu_{1}, \ldots, \mu_{p}\right)$. 
Let $\left(\tilde{\theta}_{1}, \ldots \tilde{\theta}_{p}, z_{1}, \ldots, z_{q}\right)$ be a Liouville coordinate system on $\mathcal{U}(N)$ such that $\frac{\partial}{\partial \tilde{\theta}_{i}}=$ $Z_{i}$. The 2 -form

$$
\beta=\omega-\sum_{i=1}^{p} d \mu_{i} \wedge d \tilde{\theta}_{i}
$$

is $\mathbb{T}^{p}$-invariant, and $\left.Z_{i}\right\lrcorner \beta=0 \forall i=1, \ldots, p$, hence $\beta$ is a basic 2-form with respect to the $\mathbb{T}^{p}$-action, i.e., we can write $\beta$ as (the pull-back of) a 2 -form on the space of Liouville tori:

$$
\beta=b_{i j}(z) d z_{i} \wedge d z_{j}
$$

(There is no $\tilde{\theta}_{i}$ in the expression). Thus we get the following normal form, similar to the one obtained by Fassò and Sansonetto in 2007 [25]:

$$
\omega=\sum_{i=1}^{p} d \mu_{i}(z) \wedge d \tilde{\theta}_{i}+\sum_{1 \leq i<j \leq q} b_{i j}(z) d z_{i} \wedge d z_{j} .
$$

In the above normal form, the coordinates $\left(\mu_{1}, \tilde{\theta}_{1}, \ldots, \mu_{p}, \tilde{\theta}_{p}\right)$ may be viewed as a kind of partial action-angle variables. Remark that, when $\omega$ is degenerate, the functions $\mu_{1}, \ldots, \mu_{p}$ are not functionally independent in general (see the subsection about the presymplectic situation below).

When $\omega$ is nondegenerate (it is called an almost-symplectic form in this case) then the linear independence of $Z_{1}, \ldots, Z_{p}$ implies the functional independence of $\mu_{1}, \ldots, \mu_{p}$, and so we may choose $z_{1}=\mu_{1}, \ldots, z_{p}=\mu_{p}$, and the normal form (3.5) becomes

$$
\omega=\sum_{i=1}^{p} d z_{i} \wedge d \tilde{\theta}_{i}+\sum_{1 \leq i<j \leq q} b_{i j}(z) d z_{i} \wedge d z_{j} .
$$

In this nondegenerate (but not necessarily closed) case, we may call $\left(z_{1}, \tilde{\theta}_{1}, \ldots, z_{p}, \tilde{\theta_{p}}\right)$ (generalized partial) action-angle variables, and the coordinates $\left(z_{p+1}, \ldots, z_{q}\right)$ (if there are any, i.e. if $q>p)$ are additional variables. The 2 -form $\beta=\sum_{1 \leq i<j \leq q} b_{i j}(z) d z_{i} \wedge d z_{j}$ is a kind of magnetic term (which is not necessarily closed).

Under some additional conditions on the system or on the 2 -form $\omega$, starting from the normal forms (3.5) and (3.6) we will get more refined normal forms.

\subsection{Action-angle variables for Liouville-integrable systems on (almost) sym-} plectic manifolds. When $p=q=\operatorname{dim} M / 2$ and the 2 -form $\omega$ is nondegenerate, the normal form (3.6) reads

$$
\omega=\sum_{i=1}^{n} d z_{i} \wedge d \tilde{\theta}_{i}+\sum_{1 \leq i<j \leq n} b_{i j}(z) d z_{i} \wedge d z_{j},
$$

in a coordinate system of generalized action-angle variables $\left(z_{1}, \tilde{\theta_{1}}, \ldots, z_{n}, \tilde{\theta_{n}}\right)$ on $M^{2 n}$. (We say "generalized" because there is still the magnetic term $\beta=\sum_{1 \leq i<j \leq n} b_{i j}(z) d z_{i} \wedge$ 
$\left.d z_{j}\right)$. The Liouville torus action is generated by $\left(\frac{\partial}{\partial \tilde{\theta}_{1}}, \ldots, \frac{\partial}{\partial \tilde{\theta}_{n}}\right)$ in this coordinate system.

If, moreover, $\omega$ is closed (i.e. it is really a symplectic form), then the magnetic term $\beta=\sum_{1 \leq i<j \leq q} b_{i j}(z) d z_{i} \wedge d z_{j}$ in (3.7) is also closed, $d \beta=0$, hence locally exact by Poincaré's lemma, i.e., we can write $\beta=d\left(\sum a_{i}(z) d z_{i}\right)$. It follows that $\omega=\sum d z_{i} \wedge$ $d \tilde{\theta}_{i}-\sum d z_{i} \wedge d a_{i}(z)$, i.e., we have:

$$
\omega=\sum_{i=1}^{n} d z_{i} \wedge d \theta_{i},
$$

where $\theta_{i}=\tilde{\theta}_{i}-a_{i}$.

It means that $\left(z_{1}, \theta_{1}, \ldots, z_{n}, \theta_{n}\right)$ is a system of action-angle variables (in a neighborhood $\mathcal{U}(N)$ of an arbitrary given Liouvile torus $N$ ), and the first integrals $F_{1}, \ldots, F_{n}$ of the system in $\mathcal{U}(N)$ depend only on the variables $\left(z_{1}, \ldots, z_{n}\right)$ because $X_{i}\left(F_{j}\right)=0$ $\forall i, j$. We have completed the proof of the classical Arnold-Liouville-Mineur theorem.

Remark 3.3. We may compare the present proof of existence of action-angle variables in the symplectic case with the one given in [4] and other books. There, given a Liouville system of coordinates, an analysis through Poisson brackets of the way to make standard the symplectic form $\omega$ leads to looking for an appropriate coordinate change of the form $(z, \tilde{\theta}) \mapsto(\mu(z), \tilde{\theta}-a(z))$ (our notations). The action functions $\mu_{i}$ are usually defined via the integral formula (5.17), and then one shows that the Hamiltonian vector fields of these action functions are equal to $\frac{\partial}{\partial \theta_{i}}$ by using intricate computations (which work but it's still somewhat of a mistery why they work) and/or clever geometric arguments (e.g., identifying the universal covering of a tubular neighborhood of the Liouville torus $N$ with the cotangent bundle of a local section to the torus fibration, and identifying the preimages of the section in this covering with the graphs of closed 1-forms over the section in the cotangent bundle picture, using general results from symplectic geometry). In the present proof, everything is natural: both the actions and the angles become a natural byproduct of the Hamiltonianity of the Liouville torus action: $\mu$ is its momentum map while the rotation $\tilde{\theta} \rightarrow \theta=\tilde{\theta}-a(z)$, which makes the section $\{\theta=$ const. $\}$ of the Liouville torus fibration Lagrangian (when $\omega$ is closed) is the contribution of the magnetic term which appears in the normal form for the most general 2 -form $\omega_{i}$.

3.2. Super-integrable systems on symplectic manifolds. Consider now the case when $\omega$ is a symplectic form and the integrable system $\left(X_{1}, \ldots, X_{p}, F_{1}, \ldots, F_{q}\right)$ is Hamiltonian on $(M, \omega)$, but with $p<n=\frac{1}{2} \operatorname{dim} M$ and $q=2 n-p>n$. (According to (3.4), we cannot have $p>n$ ). Such systems are often called super-integrable in the literature, because there are more first integrals than in the Liouville-integrable case.

A particularly important class of super-integrable systems are the so-called noncommutatively integrable systems introduced by Fomenko and Mischenko [53] in 1978, 
which also appeared in a book by Abraham and Marsden [1, Exercise 5.2I] at around the same time.

Action-angle variables for super-integrable Hamiltonian systems were studied by Nekhoroshev [54]. His main result says that, in this case, the symplectic form $\omega$ has the following normal form:

$$
\omega=\sum_{i=1}^{n} d \mu_{i} \wedge d \theta_{i}+\sum_{i=1}^{n-p} d x_{i} \wedge d y_{i}
$$

in a coordinate system $\left(\theta_{1}(\bmod 1), \mu_{1}, \ldots, \theta_{p}(\bmod 1), \mu_{p}, x_{1}, y_{1}, \ldots, x_{n-p}, y_{n-p}\right)$.

Formula (3.9) is of course a particular case of Formula 3.6. In order to obtain Formula (3.9), one chooses a coisotropic section $S$ to the Liouville torus fibration in $\mathcal{U}(N)$ and choose the coordinates $\theta_{i}$ such that $Z=\frac{\partial}{\partial \theta_{i}}$ and $\theta_{i}=0$ on $S$. Then $\beta=\omega-\sum_{i=1}^{n} d \mu_{i} \wedge$ $d \theta_{i}$ is $\mathbb{T}^{p}$-invariant and its pull-back to $S$ is a closed basic 2 -form with respect to the isotropic foliation on $S$. (This isotropic foliation is generated by the Hamiltonian vector fields of $\left.\theta_{1}, \ldots, \theta_{p}\right)$. On the quotient space of $S$ by the isotropic foliation, $\beta$ becomes nondegenerate, and hence can be written as $\beta=\sum_{i=1}^{n-p} d x_{i} \wedge d y_{i}$ by Darboux's theorem. These coordinates $\left(x_{i}, y_{i}\right)$ can be pulled back to $S$ and then extended to $\mathcal{U}(N)$ in a $\mathbb{T}^{p}$-invariant way, and we get a coordinates system $\left(\theta_{1}(\bmod 1), \mu_{1}, \ldots, \theta_{1}\right.$ $\left.(\bmod p), \mu_{p}, x_{1}, y_{1}, \ldots, x_{n-p}, y_{n-p}\right)$ in which $\omega$ is given by Formula (3.9).

3.3. Presymplectic action-angle variables. Consider now the case when $\omega$ is a presymplectic structure (i.e., $d \omega=0$ ) of constant rank, and $p$ is maximal possible, i.e.

$$
p=\operatorname{dim} M-\frac{1}{2} \operatorname{rank} \omega \text { and } q=\frac{1}{2} \operatorname{rank} \omega .
$$

Such a situation can happen quite often in practice. For example, starting from a Liouville-integrable Hamiltonian system on a symplectic manifold $M^{2 n}$, one fixes some regular values of some first integrals $F_{1}, \ldots, F_{d}$. Then one gets a presymplectic manifold

$$
\hat{M}=\left\{x \in M \mid F_{1}(x)=c_{1}, \ldots, F_{d}(x)=c_{d}\right\},
$$

whose presymplectic form $\omega$ (which is the pull-back of the symplectic form from $M$ ) has rank $2 n-d$, and the restricted integrable Hamiltonian system on it with $p=n, q=$ $n-d$.

By the same arguments as in the Liouville-integrable case on symplectic manifolds, one sees that in this regular presymplectic case, the presymplectic form still has the form

$$
\omega=\sum_{i=1}^{p} d \mu_{i} \wedge d \theta_{i}
$$

in a over-determined action-angle coordinate system

$$
\left(\theta_{1} \quad(\bmod 1), \mu_{1}, \ldots, \theta_{p} \quad(\bmod 1), \mu_{p}\right)
$$


on $\mathcal{U}(N) \cong \mathbb{T}^{p} \times B^{q}$ : the functions $\mu_{1}, \ldots, \mu_{p}$ are functionally dependent on $B^{q}$ and together they form a local embedding from the $q$-dimensional ball $B^{q}$ to $\mathbb{R}^{p}$.

Recall that, in the Liouville-integrable symplectic case, the action functions are uniquely determined by the system up to an integral affine transformation, and they equip the (regular part of the) base space, i.e., the space of Liouville tori, with an integral affine structure, see, e.g., [17, 22, 71]. Similarly, in the presymplectic case, the action functions are also uniquely determined by the system up to an integral affine transformation. However, since they are over-determined (i.e. functionally dependent) coordinates, they do not equip the base space (i.e., the space of Liouville tori) of the system with an integral affine structure in the usual sense, but rather with what we will call an integral co-affine structure. To be more precise, let us make the following definition.

Definition 3.4. A co-affine chart of order $p$ on a manifold $Q$ is a chart on a ball $B \subset Q$ together with an injective map $\mathcal{A}: B \rightarrow \mathbb{R}^{p}$. An (integral) co-affine structure of order $p$ on a manifold $Q$ is an atlas $Q=\cup_{i} B_{i}$ of affine charts $\left(B_{i} \subset\right.$ $Q, \mathcal{A}_{i}: B_{i} \rightarrow \mathbb{R}^{p}$ ) such that for any two chart $B_{i}$ and $B_{j}$ there is an (integral) affine transformation $T_{i j}: \mathbb{R}^{p} \rightarrow \mathbb{R}^{p}$ such that $\mathcal{A}_{j}=T_{i j} \circ \mathcal{A}_{i}$ on the intersection $B_{i} \cap B_{j}$.

Corollary 3.5. Let $N$ be a Liouville torus of an integrable Hamiltonian system of type $(p, q)$ on a presymplectic manifold $(M, \omega)$ of constant rank $2 q(q<p)$. Then the base space (i.e., space of Liouville tori) of the system in a tubular neighborhood $\mathcal{U}(N)$ of $N$ is naturally equipped with an integral co-affine structure induced by the system.

We observe that, similarly to Riemannian structures, co-affine structures have a lot of local invariants. In particular, one can talk about the local convexity, the curvature of a co-affine structure, and so on.

\section{ACtion-Angle VARIABles on CONTACT MANifolds}

One of the research problems posed by V.I. Arnold in 1995, as listed in the book Arnold's Problems [2], was to extend the theory of integrable Hamiltonian systems, in particular the theorem on action-angle variables, to contact manifolds.

In fact, before Arnold posed his problem, several authors, including Libermann [44] in 1991 and Banyaga and Molino [5] in 1992, already started the study of integrable systems on contact manifolds. Later on many other authors also worked on this and related problems, see, e.g., Lerman [41], Webster [63], Miranda [51], Khesin and Tabachnikov [37, Boyer [10, Jovanovic and Jovanovic [32, 33], etc.

In this section we will explain how to extend the classical theorem on action-angle variables to contact manifolds. But let us first recall the notion of Hamiltonian systems on contact manifolds, and introduce a natural notion of contact integrable systems.

4.1. Hamiltonian systems on contact manifolds. Recall that a contact structure on a manifold $M$ of dimension $2 n+1$ is a regular corank-1 tangent distribution $\xi$ on $M$ such that locally (in the neighborhood of every point) there is a differential 1 -form $\alpha$ such that $\xi=\operatorname{ker} \alpha$ is the kernel distribution of $\alpha$, and which satisfies the 
following nondegeneracy condition:

$$
\alpha \wedge(d \alpha)^{n} \neq 0
$$

everywhere, which means that $d \alpha$ is nondegenerate on the distribution $\xi=\operatorname{ker} \alpha$. Such an 1-form $\alpha$ is called a contact 1-form.

If one multiplies a contact 1-form by an arbitrary non-vanishing function then one gets another contact 1 -form for the same contact structure. Still, it may happen that a contact structure does not admit a global contact 1-form, due to orientation problems.

Suppose now that we have a global contact 1-form $\alpha$. The unique vector field $Z$ such that $Z\lrcorner d \alpha=0$ and $\langle\alpha, Z\rangle=1$ is called the Reeb vector field of the contact form $\alpha$. This vector field is transverse to the contact distribution $\xi=\operatorname{ker} \alpha$ and is structure-preserving, i.e. $\mathcal{L}_{Z} \alpha=0$.

Given a function $\phi$ on $M$ which is $Z$-invariant, i.e.

$$
Z(\phi)=0,
$$

there is a unique vector field $X_{\phi}$ determined by the following conditions:

$$
\left\langle\alpha, X_{\phi}\right\rangle=\phi
$$

(or equivalently, $\left\langle\alpha, X_{\phi}-\phi Z\right\rangle=0$, i.e. $X_{\phi}-\phi Z$ lies in $\xi$ ), and

$$
\left.\left.X_{\phi}\right\lrcorner d \alpha=\left(X_{\phi}-\phi Z\right)\right\lrcorner d \alpha=-d \phi .
$$

The above two equations determine $X_{\phi}-\phi Z$, and hence $X_{\phi}$, uniquely by $\phi$, because of the nondegeneracy of $\alpha$ on $\xi$. This vector field $X_{\phi}$ is called the Hamiltonian vector field of $\phi$ with respect to the contact form $\alpha$. One can check immediately that $X_{\phi}$ preserves both $\phi$ and $\alpha$, similarly to Hamiltonian vector fields on symplectic manifolds:

$$
X_{\phi}(\phi)=0 ; \mathcal{L}_{X_{\phi}} \alpha=0 .
$$

Indeed, we have $X_{\phi}(\phi)=d \alpha\left(X_{\phi}, X_{\phi}\right)=0$ and $\left.\mathcal{L}_{X_{\phi}} \alpha=X_{\phi}\right\lrcorner d \alpha+d\left\langle X_{\phi}, \alpha\right\rangle=(-d \phi)+$ $d \phi=0$.

\subsection{Integrable systems on contact manifolds.}

Definition 4.1. An integrable system $\left(X_{1}, \ldots, X_{p}, F_{1}, \ldots, F_{q}\right)$ on a contact manifold $(M, \xi)$ is called a contact integrable system on $(M, \xi)$ if the vector fields $X_{1}, \ldots, X_{p}$ preserve the contact distribution $\xi$.

Proposition 4.2. If $\left(X_{1}, \ldots, X_{p}, F_{1}, \ldots, F_{q}\right)$ is a contact integrable system on a contact manifold $(M, \xi)$ then

$$
p \leq n+1=(\operatorname{dim} M+1) / 2
$$

and in a neighborhood $\mathcal{U}(N)$ of any Liouville torus $N$ on $M$ there exists a $\mathbb{T}^{p}$-invariant contact 1 -form $\alpha$ on $\mathcal{U}(N) \cong \mathbb{T}^{p} \times B^{q}$ with a Liouville coordinate system $\left(\theta_{i}(\bmod 1), z_{j}\right)$ such that $\xi=\operatorname{ker} \alpha$ and

$$
\alpha=\sum_{1}^{p} a_{i}(z) d \theta_{i}+\sum_{1}^{q} b_{i}(z) d z_{i} .
$$


Proof. The fundamental conservation property (Theorem 2.6) means that the Liouville $\mathbb{T}^{p}$-action preserves $\xi$. Define $\alpha$ (such that $\xi=\operatorname{ker} \alpha$ ) on a section to the torus fibration and then extend it on $\mathcal{U}(N)$ via the action of $\mathbb{T}^{p}$, we get a $\mathbb{T}^{p}$-invariant contact 1 -form $\alpha$. We must have $p \leq n+1$, otherwise $\alpha \wedge(d \alpha)^{n}=0$ because it does not contain components $\left(d \theta_{1} \wedge \ldots \wedge d \theta_{p}\right) \wedge \ldots$

4.3. The generic case. Generically, the contact distribution $\xi$ is transversal to the tangent spaces of the Liouville torus $N$ (at some point, hence at every point of $N$, because of the $\mathbb{T}^{p}$-invariance):

$$
\xi \pitchfork\left(\operatorname{Span}\left(X_{1}, \ldots, X_{p}\right)=\operatorname{Span}\left(\frac{\partial}{\partial \theta_{1}}, \ldots, \frac{\partial}{\partial \theta_{p}}\right)\right) .
$$

Without loss of generality, we may assume that $\xi \pitchfork \frac{\partial}{\partial \theta_{p}}$. Dividing $\alpha$ by $\alpha\left(\frac{\partial}{\partial \theta_{p}}\right)$, we may also assume that $\alpha\left(\frac{\partial}{\partial \theta_{p}}\right)=1$, and so we get

$$
\alpha=d \theta_{p}+\sum_{1}^{p-1} a_{i}(z) d \theta_{i}+\sum_{1}^{q} b_{i}(z) d z_{i} .
$$

Notice that in this case $\frac{\partial}{\partial \theta_{p}}=Z_{\alpha}$ is the Reeb vector field of $\alpha$ :

$$
\left.\left.\left.\frac{\partial}{\partial \theta_{p}}\right\lrcorner \alpha=1, \quad \frac{\partial}{\partial \theta_{p}}\right\lrcorner d \alpha=\mathcal{L}_{\frac{\partial}{\partial \theta_{p}}} \alpha-d\left(\frac{\partial}{\partial \theta_{p}}\right\lrcorner \alpha\right)=0 .
$$

By forgetting about the coordinate $\theta_{p}$, i.e. by a natural projection from $\mathcal{U}(N)$ to $\mathbb{T}^{p-1} \times B^{q}=\mathcal{U}(N) / \mathbb{T}_{p}^{1}$, we get a Hamiltonian action of $\mathbb{T}^{p-1}$ generated by $\left(\frac{\partial}{\partial \theta_{1}}, \ldots, \frac{\partial}{\partial \theta_{p-1}}\right)$ and momentum map $\left(a_{1}, \ldots, a_{p-1}\right)$ with respect to the symplectic form $d \alpha$.

Recall that the functions $a_{i}$ in Formula (4.7) depend only on the variables $z$, and in particular they are constant on the Liouville torus $N$. Denote by $c_{i}=a_{i}(N)$ their values on $N$ (so the $c_{i}$ are constants), and let

$$
\left(\theta_{1}(\bmod 1), \ldots, \theta_{p-1} \quad(\bmod 1), z_{1}, \ldots, z_{p-1}, x_{1}, y_{1}, \ldots, x_{r}, y_{r}\right)
$$

be action-angle variables for this Hamiltonian torus action (after a change of variables $\theta_{1}, \ldots, \theta_{p-1}, z_{1}, \ldots, z_{p-1}$ if necessary), where $z_{i}=a_{i}-c_{i}$ and $r=(q+1-p) / 2$. We can write

$$
d \alpha=\sum_{1}^{p-1} d z_{i} \wedge d \theta_{i}+\sum_{1}^{r} d x_{i} \wedge d y_{i}
$$

Hence

$$
\alpha=d \theta_{p}+\sum_{1}^{p-1}\left(z_{i}+c_{i}\right) d \theta_{i}+\sum_{1}^{r} x_{i} d y_{i}+\gamma,
$$

where $\gamma$ is a closed 1 -form which is basic with respect to the $\mathbb{T}^{p}$-action. 
Put $\gamma=d f$, where $f$ is constant on each Liouville torus, and put $\theta_{0}=\theta_{p}+f$, we obtain the following normal form for $\alpha$ :

$$
\alpha=d \theta_{0}+\sum_{1}^{p-1}\left(z_{i}+c_{i}\right) d \theta_{i}+\sum_{1}^{r} x_{i} d y_{i} .
$$

Let $Y=\sum_{0}^{p-1} f_{i}(x, y, z) \frac{\partial}{\partial \theta_{i}}$ be a vector field which is constant on the tori and which preserves $\xi$. Then $\mathcal{L}_{Y} \alpha=g$. $\alpha$ where $g$ is a $\mathbb{T}^{p}$-invariant function. Moreover, we have $g=(g . \alpha)\left(\frac{\partial}{\partial \theta_{0}}\right)=\left(\mathcal{L}_{Y} \alpha\right)\left(\frac{\partial}{\partial \theta_{0}}\right)=\mathcal{L}_{Y}\left(\alpha\left(\frac{\partial}{\partial \theta_{0}}\right)\right)=Y(1)=0$, i.e. $Y$ preserves $\alpha$. Denote $\phi=\alpha(Y)$. Then $\alpha\left(Y-\phi Z_{\alpha}\right)=0$ and $\left.\left(Y-\phi Z_{\alpha}\right)\right\lrcorner d \alpha=-d \phi$, where $Z_{\alpha}=\frac{\partial}{\partial \theta_{0}}$ is the Reeb vector field of $\alpha$.

By definition, $Y$ is the Hamiltonian vector field of $\phi$ with respect to the contact 1form $\alpha$. In particular, the vector fields $X_{1}, \ldots, X_{p}$ from the beginning are Hamiltonian with respect to $\alpha$. Thus we have proved the following theorem:

Theorem 4.3 (Action-angle variables on contact manifolds). Let $\left(X_{1}, \ldots, X_{p}, F_{1}, \ldots, F_{q}\right)$ be a contact integrable system on a contact manifold $(M, \xi)$ with a Liouville torus $N \cong \mathbb{T}^{p}$ transverse to $\xi$. Then there is a contact 1 -form $\alpha$, $\operatorname{ker} \alpha=\xi$, defined in a neighborhood of $N$, which is invariant with respect to the Liouville $\mathbb{T}^{p}$-action and such that the Reeb vector field $Z_{\alpha}$ of $\alpha$ is one of the generators of this $\mathbb{T}^{p}$-action. Moreover, all the vector fields $X_{1}, \ldots, X_{p}$ are Hamiltonian with respect to $\alpha$, and there is a Liouville coordinate system

$$
\left(\theta_{0} \quad(\bmod 1), \ldots, \theta_{p-1} \quad(\bmod 1), z_{1}, \ldots, z_{p-1}, x_{1}, \ldots, x_{r}, y_{1}, \ldots, y_{r}\right)
$$

in a neighborhood $\mathcal{U}(N) \cong \mathbb{T}^{p} \times B^{q}$ of $N\left(r=(q+1-p) / 2, x_{i}, y_{i}, z_{j}\right.$ are 0 on $\left.N\right)$ in which the vector fields $X_{i}$ are constant on the Liouville tori and

$$
\alpha=d \theta_{0}+\sum_{1}^{p-1}\left(z_{i}+c_{i}\right) d \theta_{i}+\sum_{1}^{r} x_{i} d y_{i},
$$

where $c_{1}, \ldots, c_{p-1}$ are constants.

Remark 4.4. The above theorem is an improvement of the results by Banayaga and Molino [5] (the case with $p=n+1, q=n$ ) and by Jovanovic [32] (who required more conditions and obtained a less precise normal form).

In particular, in the case $p=n+1$ (maximal possible) and $q=n$, the normal form becomes

$$
\alpha=d \theta_{0}+\sum_{1}^{n}\left(z_{i}+c_{i}\right) d \theta_{i}
$$


in a coordinate system $\left(\theta_{0}(\bmod 1), \ldots, \theta_{n}(\bmod 1), z_{1}, \ldots, z_{n}\right)$, and the vector fields $X_{i}$ are of the type (with $\phi_{i}=\alpha\left(X_{i}\right)$ )

$$
X_{i}=Y_{\phi_{i}}=\left(\phi_{i}-\sum_{j=1}^{n}\left(z_{j}+c_{j}\right) \frac{\partial \phi_{i}}{\partial z_{j}}\right) \frac{\partial}{\partial \theta_{0}}+\sum_{j=1}^{n} \frac{\partial \phi_{i}}{\partial z_{j}} \frac{\partial}{\partial \theta_{j}} .
$$

4.4. The non-transversal case. As far as we know, the non-transversal case of contact integrable Hamiltonian systems had not been treated by any author in the literature.

In this case, the Liouville torus $N \cong \mathbb{T}^{p}$ is isotropic, i.e. tangent to $\xi$. Therefore, $p \leq n=(\operatorname{dim} M-1) / 2$, and the Reeb vector field $Z_{\alpha}$ of $\alpha$ is not tangent to $N$. The contact form $\alpha$ is preserved by $p+1$ vector fields $Z_{\alpha}, \frac{\partial}{\partial \theta_{1}}, \ldots, \frac{\partial}{\partial \theta_{p}}$ (and also by the $\left.X_{i}\right)$.

Proceeding as in the transversal case, but with $Z_{\alpha}$ instead of the last generator $\frac{\partial}{\partial \theta_{p}}$ of the Liouville $\mathbb{T}^{p}$-action, we get the following normal form (with $r=n-p=(q-1-p) / 2$ ):

$$
\alpha=d z_{0}+\sum_{1}^{p} z_{i} d \theta_{i}+\sum_{1}^{r} x_{i} d y_{i}
$$

In particular, if $p=n$ (maximal possible) then we have

$$
\alpha=d z_{0}+\sum_{1}^{n} z_{i} d \theta_{i}
$$

The vector fields $X_{i}$ are still Hamiltonian, with Hamiltonian functions $\phi_{i}=\alpha\left(X_{i}\right)$ :

$$
X_{i}=Y_{\phi_{i}}=\left(\phi_{i}-\sum_{j=1}^{n} z_{j} \frac{\partial \phi_{i}}{\partial z_{j}}\right) \frac{\partial}{\partial z_{0}}+\sum_{j=1}^{n} \frac{\partial \phi_{i}}{\partial z_{j}} \frac{\partial}{\partial \theta_{j}} .
$$

On the other hand, $X_{i}$ does not contain $\frac{\partial}{\partial z_{0}}$, i.e. we must have

$$
\phi_{i}-\sum_{j=1}^{n} z_{j} \frac{\partial \phi_{i}}{\partial z_{j}}=0
$$

which means that each function $\phi_{i}$ is homogeneous of degree 1 in the variables $z_{1}, \ldots, z_{n}$. So these functions $\phi_{i}$ are linear (if they are smooth), the quantities $c_{i j}=\frac{\partial \phi_{i}}{\partial z_{j}}$ are constants, and the vector fields $X_{i}=\sum c_{i j} \frac{\partial}{\partial \theta_{j}}$ are constant vector fields in our normalized coordinate system (which is quite surprising). 


\section{Action-Angle variables on Dirac and Poisson manifolds}

5.1. Dirac manifolds and their submanifolds. In this subsection, let us briefly recall some basic notions about Dirac manifolds and Hamiltonian systems on them (see, e.g., [13, 15, 38], Appendix A8 of [21], and references therein). We will also write down some basic results about (co-)Lagrangian submanifolds of Dirac manifolds, which are similar to Weinstein's results on Lagrangian submanifolds of symplectic manifolds [65], and which are related to action-angle variables.

Dirac structures were first used by Gelfand and Dorfman (see, e.g., [27, 18]) in the study of integrable systems, and were formalized by Weinstein and Courant in [16, 15] in terms of involutive isotropic subbundles of the "big" bundle $T M \oplus T^{*} M$. They generalize both (pre)symplectic and Poisson structures, and prove to be a convenient setting for dealing with systems with constraints and reduction problems.

On the direct sum $T M \oplus T^{*} M$ of the tangent and the cotangent bundles of a smooth $n$-dimensional manifold $M$ there is a natural fiber-wise indefinite symmetric scalar product of signature $(n, n)$ defined by the formula

$$
\left\langle\left(X_{1}, \alpha_{1}\right),\left(X_{2}, \alpha_{2}\right)\right\rangle=\frac{1}{2}\left(\left\langle\alpha_{1}, X_{2}\right\rangle+\left\langle\alpha_{2}, X_{1}\right\rangle\right)
$$

for sections $\left(X_{1}, \alpha_{1}\right),\left(X_{2}, \alpha_{2}\right) \in \Gamma\left(T M \oplus T^{*} M\right)$. A vector subbundle $\mathcal{D} \subset T M \oplus T^{*} M$ is called isotropic if the restriction of the indefinite scalar product to it is identically zero. On the space of smooth sections of $T M \oplus T^{*} M$ there is an operation, called the Courant bracket, defined by the formula

$$
\left.\left[\left(X_{1}, \alpha_{1}\right),\left(X_{2}, \alpha_{2}\right)\right]:=\left(\left[X_{1}, X_{2}\right], \mathcal{L}_{X_{1}} \alpha_{2}-X_{2}\right\lrcorner d \alpha_{1}\right)
$$

where $\mathcal{L}$ denotes the Lie derivative. A subbundle $\mathcal{D} \subset T M \oplus T^{*} M$ is said to be closed under the Courant bracket if the bracket of any two sections of $\mathcal{D}$ is again a section of $\mathcal{D}$.

Definition 5.1. A Dirac structure on a $n$-dimensional manifold $M$ is an isotropic vector subbundle $\mathcal{D}$ of rank $n$ of $T M \oplus T^{*} M$ which is closed under the Courant bracket. If $\mathcal{D}$ is a Dirac structure on $M$ then the couple $(M, \mathcal{D})$ is called a Dirac manifold.

We will denote the two natural projections $T M \oplus T^{*} M \rightarrow T M$ and $T M \oplus T^{*} M \rightarrow$ $T^{*} M$ by $\operatorname{proj}_{T M}$ and $\operatorname{proj}_{T^{*} M}$ respectively. We will also identify $T M$ and $T^{*} M$ with the subbundles $T M \oplus 0$ and $0 \oplus T^{*} M$ in $T M \oplus T^{*} M$.

Grosso modo, a Dirac structure $\mathcal{D}$ on a manifold $M$ is nothing but a singular foliation of $M$ by presymplectic leaves: the singular characteristic distribution $\mathcal{C}=\operatorname{proj}_{T M} \mathcal{D}$ of $\mathcal{D}$ is integrable in the sense of Frobenius-Stefan-Sussmann due to the closedness condition. On each leaf $S$ of the associated singular characteristic foliation whose tangent distribution is $\mathcal{C}$ there is an induced differential 2-form $\omega_{S}$ defined by the formula

$$
\omega_{S}(X, Y)=\left\langle\alpha_{X}, Y\right\rangle
$$

where $X, Y \in \mathcal{C}_{x}=T_{x} S$ and $\alpha_{X}$ is any element of $T_{x}^{*} M$ such that $\left(X, \alpha_{X}\right) \in \mathcal{D}_{x}$. (The pairing $\left\langle\alpha_{X}, Y\right\rangle$ does not depend on the choice of $\alpha_{X}$ as long as $\left(X, \alpha_{X}\right) \in \mathcal{D}_{x}$ : if $\alpha_{X}^{\prime}$ is another choice then $\alpha-\alpha^{\prime} \in \mathcal{D}_{x} \cap T_{x}^{*} M$, which implies that $\left\langle\alpha_{X}-\alpha_{X}^{\prime}, Y\right\rangle=0$ 
due to the isotropy of $\mathcal{D}$. The skew symmetry of $\omega$ is also due to the isotropy of $\mathcal{D}$ : $\left.\omega_{S}(X, Y)+\omega_{S}(Y, X)=\left\langle\alpha_{X}, Y\right\rangle+\left\langle\alpha_{Y}, X\right\rangle=2\left\langle\left(X, \alpha_{X}\right),\left(Y, \alpha_{Y}\right)\right\rangle=0\right)$. Due to the closedness of $\mathcal{D}$, the 2 -form $\omega_{S}$ is also closed, i.e. $\left(S, \omega_{S}\right)$ is a presymplectic manifold. The Dirac structure $\mathcal{D}$ is uniquely determined by its characteristic foliation and the presymplectic forms on the leaves.

If $\operatorname{proj}_{T M}: \mathcal{D} \rightarrow T M$ is bijective then the characteristic foliation consists of just 1 leaf, i.e. $M$ itself, and $\mathcal{D}$ is simply (the graph of) a presymplectic structure $\omega$ on $M$ : $\mathcal{D}=\{(X, X\lrcorner \omega) \mid X \in T M\}$. On the other hand, if $\operatorname{proj}_{T M}: \mathcal{D} \rightarrow T^{*} M$ is bijective then $\mathcal{D}$ is (the graph of) a Poisson structure on $M$, and the 2 -forms $\omega_{S}$ are nondegenerate, i.e. symplectic. However, in general, the ranks of the maps $\operatorname{proj}_{T^{M}}: \mathcal{D} \rightarrow T M$ and $\operatorname{proj}_{T^{M}}: \mathcal{D} \rightarrow T M^{*}$ may be smaller than $n$, and may vary from point to point.

Definition 5.2. A Dirac structure $\mathcal{D}$ on $M$ is called a regular Dirac structure of bi-corank $(r, s)$ if there are two nonnegative integers $r, s$ such that $\forall x \in M$ we have

$$
\operatorname{dim}\left(\mathcal{D}_{x} \cap T_{x} M\right)=n-\operatorname{dim} \operatorname{proj}_{T^{*} M} \mathcal{D}_{x}=r
$$

and

$$
\operatorname{dim}\left(\mathcal{D}_{x} \cap T_{x}^{*} M\right)=n-\operatorname{dim} \operatorname{proj}_{T M} \mathcal{D}_{x}=s .
$$

Even if the Dirac structure $\mathcal{D}$ is non-regular, one can still talk about its bi-corank, defined to be the bi-corank of a generic point in $M$ with respect to $\mathcal{D}$. For regular Dirac structures, we have the following analog of Darboux's theorem, whose proof is essentially the same as the proof of the classical local Darboux normal form for symplectic structures.

Proposition 5.3 (Darboux for regular Dirac). Let $O$ be an arbitrary point of a $n$ manifold $M$ with a regular Dirac structure $\mathcal{D}$ of bi-corank $(r, s)$. Then $n-r-s=2 \mathrm{~m}$ for some $m \in \mathbb{Z}_{+}$, and there is a local coordinate system $\left(x_{1}, \ldots, x_{2 m}, y_{1}, \ldots, y_{r}, z_{1}, \ldots, z_{s}\right)$ in a neighborhood of $O$, such that the local characteristic foliation is of codimension $s$ and given by the local leaves

$$
\left\{z_{1}=\text { const }, \ldots, z_{s}=\text { const }\right\},
$$

and on each of these local leaves $S$ the presymplectic form $\omega_{S}$ is given by the formula

$$
\omega_{S}=\sum_{i=1}^{m} d x_{2 i-1} \wedge d x_{2 i} .
$$

In particular, if $\mathcal{D}$ is regular, then the kernel distribution given by the kernels of the presymplectic forms is regular and integrable, and gives rise to a foliation called the kernel foliation of $\mathcal{D}$. In local canonical coordinates given by Proposition 5.3, the kernel distribution is spanned by $\left(\frac{\partial}{\partial y_{1}}, \ldots, \frac{\partial}{\partial y_{r}}\right)$.

Example 5.4. Given a manifold $L$, a regular foliation $\mathcal{F}$ on $L$, and a vector bundle $V$ over $L$, put $M=T^{*} \mathcal{F} \oplus V$, where $T^{*} \mathcal{F}$ means the cotangent bundle of the foliation $\mathcal{F}$ over $L$. Then $M$ admits the following regular Dirac structure $\mathcal{D}$, which will be called the canonical Dirac structure: each leaf $S$ of the characteristic foliation is 
of the type $S=T^{*} N \oplus V_{N}=\pi^{-1}(N)$, where $N$ is a leaf of $\mathcal{F}$ and $\pi: T^{*} \mathcal{F} \oplus V \rightarrow L$ is the projection map, and the presymplectic form on $S=T^{*} N \oplus V_{N}$ is the pullback of the standard symplectic form on the cotangent bundle $T^{*} N$ via the projection map $T^{*} N \oplus V_{N} \rightarrow T^{*} N$. When $\mathcal{F}$ consists of just one leaf $L$ and $V$ is trivial then this canonical Dirac structure is the same as the (graph of the) standard symplectic structure on $T^{*} L$.

The notions of Hamiltonian vector fields and Hamiltonian group actions can be naturally extended from the symplectic and Poisson context to the Dirac context. In particular, we have:

Definition 5.5. A vector field $X$ on a Dirac manifold $(M, \mathcal{D})$ is called a Hamiltonian vector field if there is a function $H$, called a Hamiltonian function of $X$, such that one of the following two equivalent conditions is satisfied:

i) $(X, d H)$ is a section of $\mathcal{D}$ :

$$
(X, d H) \in \Gamma(\mathcal{D}) .
$$

ii) $X$ is tangent to the characteristic distribution and

$$
X\lrcorner \omega_{S}=-d\left(\left.H\right|_{S}\right)
$$

on every presymplectic leaf $\left(S, \omega_{S}\right)$ of it.

Proposition 5.6. If $X$ is a Hamiltonian vector field of a Hamiltonian function $H$ on a Dirac manifold $(M, \mathcal{D})$, then $X$ preserves the Dirac structure $\mathcal{D}$, the function $H$, and every leaf of the characteristic foliation.

Definition 5.7. A function $f$ on $(M, \mathcal{D})$ is called a Casimir function if $f$ is a Hamiltonian function of the trivial vector field, i.e. $(0, d f) \in \Gamma(\mathcal{D})$. A vector field $X$ on $(M, \mathcal{D})$ is called an isotropic vector field if it is Hamiltonian with respect to the trivial function, i.e. $(X, d 0) \in \Gamma(\mathcal{D})$, or equivalently, $X$ lies in the kernel of the induced presymplectic forms.

Notice that if $X$ is a Hamiltonian vector field of Hamiltonian function $H, Y$ is an isotropic vector field, and $f$ is a Casimir function, then $X+Y$ is also a Hamiltonian vector field of $H$, and $X$ is also a Hamiltonian vector field of $H+f$. Modulo isotropic vector fields and Casimir functions, the correspondence between Hamiltonian vector fields and Hamiltonian functions will become bijective.

Remark also that, unlike the Poisson case, not every function on a general Dirac manifold can be a Hamiltonian function for some Hamiltonian vector field. A necessary (and essentially sufficient) condition for a function $H$ to be a Hamiltonian function is that the differential of $H$ must annul the kernels of the induced presymplectic forms.

Another interesting feature of general Dirac structures is that it is easier for a dynamical system to become Hamiltonian with respect to a Dirac structure than with respect to a symplectic or Poisson structure, as the following example shows:

Example 5.8. (See [50]). A local 2-dimensional integrable vector field with a hyperbolic singularity

$$
X=h(x, y)\left(\frac{x}{a} \frac{\partial}{\partial x}-\frac{y}{b} \frac{\partial}{\partial y}\right),
$$


where $a, b$ are two coprime natural numbers is not Hamiltonian with respect to any symplectic or Poisson structure if $a+b \geq 3$, but is Hamiltonian with respect to the presymplectic structure

$$
\omega=x^{a-1} y^{b-1} d x \wedge d y .
$$

On the other hand, a local integrable vector field

$$
X=h(y) y \frac{\partial}{\partial x}
$$

is not Hamiltonian with respect to any presymplectic structure, but is Hamiltonian with respect to the Poisson structure

$$
y \frac{\partial}{\partial x} \wedge \frac{\partial}{\partial y}
$$

If an integrable vector field on a surface admits both of the above singularities then it cannot be Hamiltonian with respect to any presymplectic or Poisson structure, but may be Hamiltonian with respect to a Dirac structure.

The theory of isotropic, coisotropic, and Lagrangian submanifolds can be naturally extended from the symplectic category to the Dirac category. However, in the Dirac case, we will have to distinguish between the Lagrangian and the co-Lagrangian submanifolds (which are the same thing in the symplectic case): A Lagrangian submanifold will lie entirely on a presymplectic leaf of the Dirac structure and is maximally isotropic there. On the other hand, a co-Lagrangian submanifold will intersect the whole local family of presymplectic leaves, with each intersection being isotropic but with tangent spaces having trivial intersection with the kernels of the presymplectic form. Moreprecisely, we have:

Definition 5.9. Let $(M, \mathcal{D})$ be a Dirac manifold, where $\mathcal{D}$ is a regular Dirac structure of bi-corank $(r, s)$.

i) A submanifold $N$ of $(M, \mathcal{D})$ is called isotropic if it lies on a characteristic leaf $S$, and the pull-back of the presymplectic form $\omega_{S}$ to $N$ is trivial. If, moreover, $N$ is of maximal dimension possible, i.e.

$$
\operatorname{dim} N=\frac{1}{2} \operatorname{rank} \omega_{S}+r=\frac{1}{2}(\operatorname{dim} M+r-s),
$$

then $N$ is called a Lagrangian submanifold. A foliation (or fibration) on $(M, \mathcal{D})$ is called Lagrangian if its leaves (or fibers) are Lagrangian.

ii) A submanifold $L$ of $(M, \mathcal{D})$ is called a co-Lagrangian submanifold if

$$
\operatorname{dim} L=\frac{1}{2}(\operatorname{dim} M-r+s),
$$

and for every point $x \in L$ the tangent space $T_{x} L$ satisfies the following conditions : a) $T_{x} L+\operatorname{proj}_{T M} \mathcal{D}_{x}=T_{x} M$; b) $\left.T_{x} L \cap\left(T_{x} M \cap \mathcal{D}_{x}\right)=\{0\} ; c\right)\left.\omega_{S}\right|_{T_{x} L}=0$ where $S$ is the characteristic leaf containing $x$.

Observe that if $N$ is a Lagrangian submanifold then $T_{x} N$ contains the kernel of the presymplectic form at $x$ for every $x \in N$, this kernel distribution is regular and integrable in $N$, and $N$ is foliated by the kernel foliation. If $L$ is a co-Lagrangian 
submanifold then $L$ is also foliated: the foliation on $L$ is the intersection of the characteristic foliation of $(M, \mathcal{D})$ with $L$. Moreover, if $N$ is a Lagrangian submanifold and $L$ is a co-Lagrangian submanifold of $(M, \mathcal{D})$, then

$$
\operatorname{dim} N+\operatorname{dim} L=\operatorname{dim} M
$$

The above definition of Lagrangian and co-Lagrangian submanifolds may differ from the other definitions in the literature, but they are well-suited for our study of actionangle variables. In particular, it is easy to see that any local Lagrangian foliation in a regular Dirac manifold admits a local co-Lagrangian section. We also have the following analogs of some results of Weinstein [65] about (co-)Lagrangian submanifolds:

Theorem 5.10 (Co-Lagrangian embeddings). Let $L$ be a co-Lagrangian submanifold of a regular Dirac manifold $(M, \mathcal{D})$. Then there is a foliation $\mathcal{F}$ on $L$, a vector bundle $V$ over $L$, and a Dirac diffeomorphism from a neighborhood $(\mathcal{U}(L), \mathcal{D})$ of $L$ to an open subset of $T^{*} \mathcal{F} \oplus V$ equipped with the canonical Dirac structure, which sends $L$ to the zero section of $T^{*} \mathcal{F} \oplus V$.

Proof. Let us first prove the above theorem in the Poisson case: $\mathcal{D}=\{(\alpha\lrcorner \Pi, \alpha) \mid \alpha \in$ $\left.T^{*} M\right\}$ is the graph of a regular Poisson structure $\Pi$ on $M$. In this case, the bi-corank of $\mathcal{D}$ is of the type $(0, s)$, and the leaves of the characteristic foliation are symplectic.

Denote by $\mathcal{F}$ the foliation on $L$, which is the intersection of the characteristic foliation with $L: T_{x} \mathcal{F}=T_{x} N \cap \mathcal{C}_{x} F$ for every $x \in N$, where $\mathcal{C}$ is the characteristic distribution. Then, via the symplectic form on $\mathcal{C}$, the vector bundle $T^{*} \mathcal{F}$ over $L$ is naturally isomophic to another vector bundle over $L$, whose fiber over $x \in L$ is the quotient space $\mathcal{C}_{x} / T_{x} \mathcal{F}$. This latter bundle is also naturally isomorphic to the normal bundle of $L$ in $M$. Due to these isomorphisms, there is a vector subbundle $E$ over $L$ of $\mathcal{C}_{L}=\cup_{x \in L} \mathcal{C}_{x}$, such that $\mathcal{C}_{L}=T \mathcal{F} \oplus E$, and $E$ is Lagrangian, i.e. $E$ is isotropic with respect to the induced symplectic forms on $\mathcal{C}$ and the rank of $E$ is half the rank of $\mathcal{C}_{L}$.

At each point $x \in L$, the set of germs of local Lagrangian submanifolds in $M$ which contain $x$ and which are tangent to $E_{x}$ at $x$ is a contractible space. (By a local symplectomorphism from the characteristic leaf $S$ which contains $x$ to $T^{*} \mathbb{R}^{m}$ where $2 m=\operatorname{rank} \omega_{S}$, this space of germs can be identified with the space of germs of exact 1 -forms on $\left(\mathbb{R}^{m}, 0\right)$ whose 1 -jets vanish at the origin). Due to this fact, there are no topological obstructions to the existence of a Lagrangian foliation in a sufficiently small neighborhood of $L$ which is tangent to $E_{x}$ at every point $x \in L$. Denote by $\mathcal{N}$ such a Lagrangian foliation. Identify $L$ with the zero section of $T^{*} \mathcal{F}$. Then, similarly to the proof of uniqueness of marked symplectic realizations of Poisson manifolds (see Proposition 1.9.4 of [21]), one can show that there is a unique Poisson isomorphism $\Phi$ from a neighborhood of $N$ in $M$ to a neighborhood of $L$ of in $T^{*} \mathcal{F}$, which is identity on $L$ and which sends the leaves of $N$ to the local fibers of $T^{*} \mathcal{F}$. $\Phi$ can be constructed as follows:

Take a local function $F$ in the neighborhood of a point $x \in L$ in $M$, which is invariant on the leaves of the Lagrangian foliation $\mathcal{N}$. Push $F$ to $T^{*} \mathcal{F}$ by identifying $L$ with the zero section of $T^{*} \mathcal{F}$ and by making the function invariant (i.e., constant) on the fibers of $T^{*} \mathcal{F}$. Denote the obtained local function on $T^{*} \mathcal{F}$ by $\tilde{F}$. Now extend the map 
$\Phi$ from $L$ (on which $\Phi$ is the identity map) to a neighborhood of $L$ by the flows of the Hamiltonian vector fields $X=X_{F}$ and $\tilde{X}=X_{\tilde{F}}$ of $F$ and $\tilde{F}$ : if $y=\phi_{X}^{t}(z)$ where $z \in L$ and $\phi_{X}^{t}$ denotes the time- $t$ flow of $X$, then $\Phi(y)=\phi_{\tilde{X}}^{t}(z)$. One verifies easily that $\Phi$ is well-defined (i.e. it does not depend on the choice of the functions $F$ ), and is a required Poisson isomorphism.

Consider now the general regular Dirac case. Denote by $\mathcal{K}$ the kernel foliation in a small tubular neighborhood $\mathcal{U}(L)$ of $L$ in $M$ in this case: the tangent space of $\mathcal{K}$ at each point is the kernel of the induced presymplectic form at that point. Denote by $\mathcal{M} \subset \mathcal{U}(L)$ a submanifold which contains $N$ and which is transversal to the kernel foliation. Then $\mathcal{M}$ is Poisson submanifold of $(M, \mathcal{D})$. Denote by $\pi_{1}: \mathcal{U}(L) \rightarrow \mathcal{M}$ the projection map (whose preimages are the local leaves of the kernel foliation). The Dirac structure $\mathcal{D}$ in $\mathcal{U}(L)$ is uniquely obtained from the Poisson structure on $\mathcal{M}$ by pulling back the symplectic 2 -forms from the characteristic leaves of $\mathcal{M}$ to the characteristic leaves of $\mathcal{U}(L)$ via the projection map $\pi_{1}$ (so that they become presymplectic with the prescribed kernels). Denote by $V$ the vector bundle over $L$ which is the restriction of the kernel distribution to $L$.

According to the Poisson case of the theorem, there is a Poisson diffeomorphism from $\mathcal{M}$ to a neighborhood of the zero section in $T^{*} \mathcal{F}$. Extend $\Phi$ to an arbitrary diffeomorphism $\hat{\Phi}$ from $\mathcal{U}(L)$ to a neighborhood of the zero section in $T^{*} \mathcal{F} \oplus V$ which is fiber-preserving in the sense that $\pi_{2} \circ \hat{\Phi}=\Phi \circ \pi_{1}$, where $\pi_{2}$ denotes the projection $T^{*} \mathcal{F} \oplus V \rightarrow T^{*} \mathcal{F}$. Then $\hat{\Phi}$ is a required Dirac diffeomorphism.

Theorem 5.11 (Co-Lagrangian sections). Let $\mathcal{F}$ be a regular foliation on a manifold $L$, and $V$ be a vector bundle over $L$. Then a section $K$ of the vector bundle $T^{*} \mathcal{F} \oplus V$ equipped with the canonical Dirac structure is a co-Lagrangian submanifold of $T^{*} \mathcal{F} \oplus V$ if and only if $K=(\theta, v)$, where $\theta \in \Gamma\left(T^{*} \mathcal{F}\right)$ with $d_{\mathcal{F}} \theta=0$, and $v \in \Gamma(V)$ is arbitrary.

Proof. The proof is the same as in the symplectic case, when $V$ is trivial and $\mathcal{F}$ consists of just one leaf, i.e. $L$ itself.

5.2. Integrable Hamiltonian systems on Dirac manifolds. The following natural definition is a straightforward generalization of the notion of integrable Hamiltonian systems from the case of pre-symplectic and Poisson manifolds to the case of general Dirac manifolds.

Definition 5.12. An integrable system $\left(X_{1} \ldots, X_{p}, F_{1}, \ldots, F_{q}\right)$ of type $(p, q)$ on a Dirac manifold $(M, \mathcal{D})$ is called an integrable Dirac system if the vector fields $X_{1}, \ldots, X_{p}$ preserve the Dirac structure $\mathcal{D}$. It is called an integrable Hamiltonian system if the vector fields $X_{1}, \ldots, X_{p}$ are Hamiltonian, i.e., there are Hamiltonian functions $H_{1}, \ldots, H_{p}$ such that $\left(X_{i}, d H_{i}\right) \in \Gamma(\mathcal{D})$ for $i=1, \ldots, p$.

Of course, an integrable Hamiltonian system on a Dirac manifold is also an integrable Dirac system, but the converse is not true.

Proposition 5.13. Let $N$ be a Liouville torus of an integrable Hamiltonian system $\left(X_{1} \ldots, X_{p}, F_{1}, \ldots, F_{q}\right)$ with corresponding Hamiltonian functions $H_{1}, \ldots, H_{p}$ on a Dirac manifold $(M, \mathcal{D})$. Then we have: 
i) The functions $H_{1}, \ldots, H_{p}$ are invariant on the Liouville tori in a tubular neighborhood $\mathcal{U}(N)$ of $N$.

ii) The Liouville tori in $\mathcal{U}(N)$ are isotropic.

iii) The functions $H_{1}, \ldots, H_{p}$ commute with each other in $\mathcal{U}(N)$, i.e. their Poisson brackets vanish: $\left\{H_{i}, H_{j}\right\}=0$.

Proof. Recall that, similarly to the case of Poisson manifolds, if $H$ and $F$ are two Hamiltonian functions on a Dirac manifold with two corresponding Hamiltonian vector fields $X_{H}$ and $X_{F}$, then their Poisson bracket $\{H, F\}:=X_{H}(F)=-X_{F}(H)=\omega_{S}\left(X_{H}, X_{F}\right)$ (where $\omega_{S}$ denotes the induced presymplectic forms) is again a Hamiltonian function whose associated Hamiltonian vector field is equal to $\left[X_{H}, X_{F}\right]$ plus an isotropic vector field.

Since $\left[X_{i}, X_{j}\right]=0$ (for any $i, j \leq p$ ) we have that $X_{i}\left(H_{j}\right)=\left\{H_{i}, H_{j}\right\}$ is a Casimir function. In particular, $X_{i}\left(H_{j}\right)$ is invariant on the Liouville tori near $N$, because the Liouville tori belong to the characteristic leaves (because the tangent bundle of the Liouville tori are spanned by the Hamiltonian vector fields $X_{1} \ldots, X_{p}$ which are tangent to the characteristic distribution). But the average of $X_{i}\left(H_{j}\right)$ on each Liouville torus is 0 due to the quasi-periodic nature of the $X_{i}$ (Theorem 2.1) (see the paragraph after Definition 3.1 for a more detailed explanation, the situation here is the same), so $X_{i}\left(H_{j}\right)=0$ on each Liouville torus, i.e., we have

$$
X_{i}\left(H_{j}\right)=\left\{H_{i}, H_{j}\right\}=0 \text { in } \mathcal{U}(N) \forall i=1, \ldots, p,
$$

which implies that $H_{j}$ is invariant on the Liouville tori for all $j=1, \ldots, p$.

The proof of the isotropicness of Liouville tori is absolutely similar to the presymplectic case: it follows from the equation $\omega_{S}\left(X_{i}, X_{j}\right)=\left\{H_{i}, H_{j}\right\}=0$ and the fact that the vector fields $X_{1}, \ldots, X_{p}$ span the tangent bundles of the Liouville tori.

5.3. Action functions. We have the following result about Hamiltonianity of the Liouville torus actions for integrable Hamiltonian systems on Dirac manifolds, similarly to the case of systems on manifolds with a differential 2-form (Proposition 3.2):

Theorem 5.14 (Liouville torus action is Hamiltonian). Let $N$ be a Liouville torus of an integrable Hamiltonian system $\left(X_{1} \ldots, X_{p}, F_{1}, \ldots, F_{q}\right)$ on a Dirac manifold $(M, \mathcal{D})$. Assume that at least one of the following two additional conditions is satisfied:

i) The dimension $\operatorname{dim}\left(\operatorname{Span}_{\mathbb{R}}\left(X_{1}(x), \ldots, X_{p}(x)\right) \cap\left(T_{x} M \cap \mathcal{D}_{x}\right)\right)$ is constant in a neighborhood of $N$.

ii) The characteristic foliation is regular in a neighborhood of $N$.

Then the Liouville torus action of the system is a Hamiltonian torus action (i.e. its generators are Hamiltonian) in a neighborhood $\mathcal{U}(N)$ of $N$.

In particular, if $\mathcal{D}$ is a Poisson structure then condition i) holds, and if $\mathcal{D}$ is a presymplectic structure then condition ii) holds, and the theorem is valid in both cases. We don't know whether the above theorem is still true in the "singular" case when both of the above two conditions fail or not: we have not been able to produce a proof nor a counter-example. 
Proof. Let us first prove the theorem under condition ii), i.e. the characteristic foliation if regular. Fix a tubular neighborhood $\mathcal{U}(N) \cong \mathbb{T}^{p} \times B^{q}$ with a coordinate system

$$
\left(\theta_{1}(\bmod 1), \ldots, \theta_{p} \quad(\bmod 1), z_{1}, \ldots, z_{q}\right)
$$

in which the vector fields $X_{1}, \ldots, X_{p}$ are constant on Liouville tori, as given by Theorem 2.1. What we need to show is that $\frac{\partial}{\partial \theta_{1}}$ is a Hamiltonian vector field. (Then, by similar arguments, the vector fields $\frac{\partial}{\partial \theta_{2}}, \ldots, \frac{\partial}{\partial \theta_{p}}$ are also Hamiltonian, so the Liouville torus action is Hamiltonian). By Theorem 2.1, we can write

$$
\frac{\partial}{\partial \theta_{1}}=\sum_{i=1}^{p} r_{i} X_{i},
$$

where the functions $r_{i}$ are invariant on the Liouville tori. Put

$$
\rho=\sum_{i=1}^{p} r_{i} d H_{i} .
$$

Then $\left(\frac{\partial}{\partial \theta_{1}}, \rho\right)=\sum_{i=1}^{p} r_{i}\left(X_{i}, d H_{i}\right) \in \Gamma(\mathcal{D})$ is a section of the Dirac structure. (If $\mathcal{D}$ is the graph of a presymplectic structure $\omega$ then $\rho$ is simply the contraction of $\omega$ with $\frac{\partial}{\partial \theta_{1}}$ ). Since, by Theorem [2.6, $\frac{\partial}{\partial \theta_{1}}$ preserves the Dirac structure, it also preserves the presymplectic structure $\omega_{S}$ of each characteristic leaf $S$, and therefore $\left.d \rho\right|_{S}=$ $\left.d\left(\frac{\partial}{\partial \theta_{1}}\right\lrcorner \omega_{S}\right)=\mathcal{L}_{\frac{\partial}{\partial \theta_{1}}} \omega_{S}=0$, i.e., the restriction of $\rho$ to each characteristic leaf is closed.

Notice that, by condition ii) and Proposition 5.13, each characteristic leaf in $\mathcal{U}(N)$ is a trivial fibration by Liouville tori over a disk. The 1 -form $\rho$ is not only closed, but actually exact, on each characteristic leaf, because its pull-back to each Liouville torus is trivial by construction and by Proposition 5.13 .

We can define a Hamiltonian function $\mu_{1}$ associated to $\frac{\partial}{\partial \theta_{1}}$ as follows (in the presymplectic case, $\mu_{1}$ is just a function whose differential is $\rho$, but here the constrution is a bit more elaborate because if we do it leaf-wise we have to assure that the end result is a smooth function):

Fix a point $x_{0} \in N$, and let $D$ be a small disk containing $x_{0}$ which is transversal to the characteristic foliation. Let $H_{1}, \ldots, H_{p}$ be arbitrary Hamiltonian functions associated to $X_{1}, \ldots, X_{p}$. For each $y \in \mathcal{U}(N)$, denote by $y_{0}$ the intersection point of the characteristic leaf through $y$ in $\mathcal{U}(N)$ with $D$, and define

$$
\mu_{1}(y)=\int_{y_{0}}^{y} \rho
$$

where the above integral means the integral of $\rho$ over a path on a characteristic leaf from $y_{0}$ to $y$. The function $\mu_{1}(y)$ is well defined, i.e. single-valued and does not depend 
on the choice of the path, because of the exactness of $\rho$ on the characteristic leaves. It is also obvious that $d \mu_{1}=\rho$, i.e. $\mu_{1}$ is a Hamiltonian function of $\frac{\partial}{\partial \theta_{1}}$.

Let us now assume that condition ii) fails, but condition i) holds, i.e.

$$
d=\operatorname{dim}\left(\operatorname{Span}_{\mathbb{R}}\left(X_{1}(x), \ldots, X_{p}(x)\right) \cap\left(T_{x} M \cap \mathcal{D}_{x}\right)\right)
$$

is a constant on $\mathcal{U}(N)$. Without loss of generality, we can assume that $X_{1}(x), \ldots, X_{p-d}(x)$ are linearly independent modulo $\left.\operatorname{Span}_{\mathbb{R}}\left(X_{1}(x), \ldots, X_{p}(x)\right) \cap\left(T_{x} M \cap \mathcal{D}_{x}\right)\right)$ for any $x \in \mathcal{U}(N)$. It implies that $d H_{1} \wedge \ldots \wedge d H_{p-d}(x) \neq 0$ everywhere in $\mathcal{U}(N)$. By the inverse function theorem, there exists a disk $D$ which intersects the characteristic leaf $S \ni x_{0}$ transversally at $x_{0}$, and such that the functions $H_{1}, \ldots, H_{p-d}$ are invariant on $D$.

Define the action function $\mu_{1}$ by the same Formula (5.16) as above, with $y_{0} \in D$. Since the characteristic foliation in $\mathcal{U}(N)$ is singular, a general characteristic leaf in $\mathcal{U}(N)$ can intersect $D$ at a submanifold instead of just a point. In order to show that $\mu_{1}$ is well-defined, we have to check that if $\gamma$ is an arbitrary oriented curve lying on the intersection of a characteristic leaf $S$ with the disk $D$, then we have $\int_{\gamma} \rho=0$. But it is the case, because the pull-back of $d H_{i}$ to $\gamma$ is trivial for all $i=1, \ldots, p$ by construction. Thus $\mu_{1}$ is a well-defined single-valued Hamiltonian function of $\frac{\partial}{\partial \theta_{1}}$, and the theorem is proved.

The Hamiltonian functions $\mu_{1}, \ldots, \mu_{p}$ of the generators $\frac{\partial}{\partial \theta_{1}}, \ldots, \frac{\partial}{\partial \theta_{p}}$ of the Liouville torus action given in Theorem 5.14 will be called action functions or action variables of the integrable system. Notice that the action functions are determined by the system only up to Casimir functions and up to a choice of the generators of the Liouville torus action (or in other words, a choice of the basis of the torus $\mathbb{T}^{p}$ ).

Remark 5.15. Another way to obtain action variables in the symplectic case is by the following classical integral formula for action functions, which was known already to Einstein and other physicists (see, e.g., 6]), and which was already used by Mineur in his proof of the existence of action-angle variables [49]:

$$
\mu_{1}=\int_{\gamma_{1}} \alpha
$$

where $\alpha$ is a 1 -form such that $\left.d \alpha\right|_{S}=\omega_{S}$, and $\gamma_{1}$ is the loop generated by the vector field $\frac{\partial}{\partial \theta_{1}}$ on the Liouville torus (for each torus). But it is not easy to use Formula (5.17) on Dirac manifolds, because of the problem of existence and regularity of $\alpha$ in the Dirac case. That's why in the proof of Theorem 5.14 we used Formula (5.16) instead of Formula (5.17) for the action functions. In the symplectic case, one can see easily that Formula (5.16) and Formula (5.17) give rise to the same action function. Indeed, by moving the path from $y_{0}$ to $y$ by the flow of $\frac{\partial}{\partial \theta_{1}}$, we get an annulus with two boundary components, one is a loop on the Liouville torus $N_{0}$ wich contains $y_{0}$ and 
the other one is a loop on the Liouville torus $N$ wich contains $y$. By Stokes theorem, the difference between the values of the action function defined by Formula (5.17) on $N$ and on $N_{0}$ is equal to the integral of the symplectic form $\omega=d \alpha$ over that annulus. On the other hand, the contraction of $\omega$ with $\frac{\partial}{\partial \theta_{1}}$ is $\rho$, and so this integral of $\omega$ over the annulus is equal to the integral in Formula (5.16).

5.4. Action-angle variables on Poisson manifolds. Recall that 2 -vector field $\Pi$ on a manifold $M$ is called a Poisson structure if the associated Poisson bracket $\{f, g\}=\langle d f \wedge d g, \Pi\rangle$ satisfies the Jacobi identity, or equivalently, $[\Pi, \Pi]=0$, where [., .] denotes the Schouten bracket, see, e.g., [21. Given a function $G$ on a Poisson manifold $(M, \Pi)$, the vector field $X=d G\lrcorner \Pi$ is called the Hamiltonian vector field of $G$ with respect to $\Pi$, and it preserves $\Pi: \mathcal{L}_{X} \Pi=0$.

An integrable system $\left(X_{1}, \ldots, X_{p}, F_{1}, \ldots, F_{q}\right)$ on a Poisson manifold $(M, \Pi)$ is called Hamiltonian with respect to $\Pi$ if all the vector fields $\left.X_{i}=d G_{i}\right\lrcorner \Pi(i=1, \ldots, p)$ are Hamiltonian. In this case, the functions $G_{i}$ are automatically invariant with respect to the Liouville $\mathbb{T}^{p}$-action in the neighborhood of each Liouville torus. Let us recall the following theorem, which is a special case of Theorem 5.14,

Theorem 5.16 (The Liouville torus action is Hamiltonian). Let $N$ be a Liouville torus of an integrable Hamiltonian system $\left(X_{1}, \ldots, X_{p}, F_{1}, \ldots, F_{q}\right)$ on a Poisson manifold $(M, \Pi)$. Then the Liouville $\mathbb{T}^{p}$-action in a neighborhood $\mathcal{U}(N)$ of $N$ is a Hamiltonian torus action.

Using Theorem 5.16 and the same arguments as in the proof of Weinstein's splitting theorem for Poisson structures (see, e.g., [21, 66]), one gets the following result about action-angle variables near a Liouville torus of an integrable Hamiltonian system on a Poisson manifold, which was obtained earlier by C. Laurent-Gengoux, E. Miranda and P. Vanhaecke [40] with a much longer proof.

Theorem 5.17 (Laurent-Miranda-Vanhaecke). Let $N$ be a Liouville torus of an integrable Hamiltonian system $\left(X_{1}, \ldots, X_{p}, F_{1}, \ldots, F_{q}\right)$ of type $(p, q)$ on a twisted Poisson manifold $(M, \Pi)$. Then $q \geq p$, and on a neighborhood $\mathcal{U}(N) \cong \mathbb{T}^{p} \times D^{q}$ of $N$ there exists a coordinate system

$$
\left(\theta_{1}(\bmod 1), \ldots, \theta_{p} \quad(\bmod 1), z_{1}, \ldots, z_{q}\right)
$$

such that

$$
\Pi=\sum_{i=1}^{p} \frac{\partial}{\partial z_{i}} \wedge \frac{\partial}{\partial \theta_{i}}+\sum_{p<i<j \leq q} b_{i j}(z) \frac{\partial}{\partial z_{i}} \wedge \frac{\partial}{\partial z_{j}}
$$

in $\mathcal{U}(N)$, the functions $F_{1}, \ldots, F_{q}$ do not depend on the variables $\theta_{1}, \ldots, \theta_{p}$, and the vector fields $X_{i}$ can be written as $X_{i}=\sum_{j} c_{i j}(z) \frac{\partial}{\partial \theta_{j}}$.

Proof. Denote by $Z_{1}, \ldots, Z_{p}$ the generators of the Liouville $\mathbb{T}^{p}$-action, which is a Hamiltonian torus action by Theorem [5.16, and denote by $\left(z_{1}, \ldots, z_{p}\right): \mathcal{U}(N) \rightarrow \mathbb{R}^{p}$ a corresponding momentum map. 
By induction, we can construct one by one the periodic functions $\theta_{1}, \theta_{2}, \ldots, \theta_{p}$ $(\bmod 1)$ on $\mathcal{U}(N)$ with the following properties:

i) $Z_{i}\left(\theta_{j}\right)=\delta_{i j}$ is the Kronecker symbol $\left(\delta_{i j}=1\right.$ if $i=j$ and $\delta_{i j}=0$ if $\left.i \neq j\right)$.

ii) $\left\{\theta_{i}, \theta_{j}\right\}_{\Pi}=0$ for all $i, j=1, \ldots, p$.

(It is equivalent to the construction of a coisotropic section $S$ to the Liouville torus fibration in $\mathcal{U}(N)$, and then put $\theta_{i}=0$ on $S$ and then extend them to $\mathcal{U}(N)$ in a natural way so that $\left.Z_{i}\left(\theta_{j}\right)=\delta_{i j}\right)$.

The functions $z_{1}, \ldots, z_{p}$ are functionally dependent and $\mathbb{T}^{p}$-invariant, so we can choose $q-p$ additional $\mathbb{T}^{p}$-invariant coordinates $z_{p+1}, \ldots, z_{q}$ to get a complete coordinate system on $\mathcal{U}(N)$. In this coordinate system $\left(\theta_{1}(\bmod 1), \ldots, \theta_{p}(\bmod 1), z_{1}, \ldots, z_{q}\right)$, all the coefficients of $\Pi$ are $\mathbb{T}^{p}$-invariant (i.e., they do not depend on the coordinates $\left.\theta_{i}\right)$; there is no term of the type $\frac{\partial}{\partial z_{i}} \wedge \frac{\partial}{\partial z_{j}}$ with $i \leq p$ or of the type $\frac{\partial}{\partial z_{i}} \wedge \frac{\partial}{\partial \theta_{j}}$ with $i \neq j$ in the expression of $\Pi$ because $\left.d z_{i}\right\lrcorner \Pi=\frac{\partial}{\partial \theta_{i}}$. There is no term of the type $\frac{\partial}{\partial \theta_{i}} \wedge \frac{\partial}{\partial \theta_{j}}$ in the expression of $\Pi$ either, because $\left\{\theta_{i}, \theta_{j}\right\}=0$. That's why $\Pi$ has the expression (5.19).

In particular, in the case of Liouville-integrable Hamiltonian systems on Poisson manifolds (i.e. the Poisson structure is regular and the dimension of the Liouville torus is half the dimension of the symplectic leaves) then the term $\sum_{p<i<j \leq q} b_{i j}(z) \frac{\partial}{\partial z_{i}} \wedge \frac{\partial}{\partial z_{j}}$ disappears, and the Poisson structure $\Pi$ has the simple form

$$
\Pi=\sum_{i=1}^{p} \frac{\partial}{\partial z_{i}} \wedge \frac{\partial}{\partial \theta_{i}}
$$

similarly to the symplectic case. In fact, this Liouville-integrable Poisson case is nothing but a parametrized version of the classical Liouville-integrable symplectic case.

5.5. Full action-angle variables on Dirac manifolds. As was shown in Proposition 5.13. Liouville tori of integrable Hamiltonian systems are isotropic. As a consequence, their dimension satisfies the inequality

$$
\operatorname{dim} N \leq \frac{1}{2} \operatorname{rank} \omega_{S}+r
$$

where $r=\operatorname{dim}\left(\mathcal{D}_{x} \cap T_{x} M\right)$ is the corank of $\omega$ on the characteristic leaf containing a Liouville torus $N$. The dimension of $N$ is the number of angle variables, and also the number of action variables that we can have.

In the optimal case, when the above inequality becomes equality, i.e., $N$ is a Lagrangian submanifold, then we will say that we have a full set of action-angle variables. The word "full" means that the presymplectic form in this case can be completely described in terms of action-angle variables. More precisely, we have:

Theorem 5.18 (Full action-angle variables). Let $N$ be a Liouville torus of an integrable Hamiltonian system

$$
\left(X_{1}, \ldots, X_{m+r}, F_{1}, \ldots, F_{m+s}\right)
$$


on a regular Dirac manifold $(M, \mathcal{D})$ of bi-corank $(r, s)$ and dimension $n=2 m+r+s$. Then the Liouville tori of the system in a tubular neighborhood $\mathcal{U}(N)$ are Lagrangian submanifolds of $(M, \mathcal{D})$, and there is a coordinate system

$$
\left(\theta_{1}(\bmod 1), \ldots, \theta_{m+r} \quad(\bmod 1), z_{1}, \ldots, z_{m+s}\right)
$$

on

$$
\mathcal{U}(N) \cong \mathbb{T}^{m+r} \times B^{m+s}
$$

and action functions

$$
\mu_{1}=z_{1}, \ldots, \mu_{m}=z_{m}, \mu_{m+1}, \ldots, \mu_{m+r}
$$

on $\mathcal{U}(N)$, such that the Liouville torus action is generated by $\left(\frac{\partial}{\partial \theta_{1}}, \ldots, \frac{\partial}{\partial \theta_{m+r}}\right)$, the functions $\mu_{m+1}, \ldots, \mu_{m+r}$ depend only on the coordinates $z_{1}, \ldots, z_{m+s}$, the characteristic leaves of $\mathcal{D}$ in $\mathcal{U}(N)$ are

$$
S_{c_{1}, \ldots, c_{s}}=\left\{z_{m+1}=c_{1}, \ldots z_{m+s}=c_{s}\right\},
$$

and the presymplectic form $\omega_{S}$ on each leaf $S=S_{c_{1}, \ldots, c_{s}}$ is

$$
\omega_{S}=\left.\left(\sum_{i=1}^{m+r} d \mu_{i} \wedge d \theta_{i}\right)\right|_{S} .
$$

Proof. The fact that the Liouville tori are Lagrangian is given by Proposition 5.13 and the definition of Lagrangian submanifolds. Since the fibration by Liouville tori is Lagrangian, we can choose a co-Lagrangian section $D$ of this fibration, and a coordinate system

$$
\left(\theta_{1} \quad(\bmod 1), \ldots, \theta_{m+r} \quad(\bmod 1), z_{1}, \ldots, z_{m+s}\right)
$$

on $\mathcal{U}(N)$ such that the leaves of the regular characteristic foliation are given by Formula (5.26) and the functions $\theta_{1}, \ldots, \theta_{m+r}$ vanish on $D$, i.e. the co-Lagrangian disk $D$ is given by the equation

$$
D=\left\{\theta_{1}=0, \ldots, \theta_{m+r}=0\right\} .
$$

The existence of action variables $\mu_{1}, \ldots, \mu_{m+r}$ corresponding to the vector fields $\frac{\partial}{\partial \theta_{1}}, \ldots, \frac{\partial}{\partial \theta_{m+r}}$ is given by Theorem 5.14. Without loss of generality, we can assume that $T N$ is spanned by $\frac{\partial}{\partial \theta_{1}}, \ldots, \frac{\partial}{\partial \theta_{m}}$ and the kernel $K=\mathcal{D} \cap T M$. Then

$$
\left.d \mu_{1} \wedge \ldots \wedge d \mu_{m}\right|_{S} \neq 0
$$

everywhere in $\mathcal{U}(N)$, i.e., the functions $\mu_{1}, \ldots, \mu_{m}$ are functionally independent on the symplectic leaves, but

$$
\left.d \mu_{1} \wedge \ldots \wedge d \mu_{m} \wedge d \mu_{m+i}\right|_{S}=0 \forall i=1, \ldots, r .
$$

It follows that we can put $z_{1}=\mu_{1}, \ldots, z_{m}=\mu_{m}$, and choose $z_{m+1}, \ldots, z_{m+s}$ to be Casimir functions. 
It remains to prove Formula (5.27). By the invariance of everything with respect to the Liouville torus action, it is enough to prove this formula at a point $x \in D$. Without loss of generality, we can assume that $\{x\}=N \cap D$.

If $X, Y \in T_{x} S$ are two vector fields tangent to the characteristic foliation at $x$ such that $X, Y \in T_{x} N$, then $\omega_{S}(X, Y)=0$ due to the isotropy of $N$, and $d \mu_{i}(X)=d \mu_{i}(Y)=$ 0 for all $i=1, \ldots, m+r$, which implies that $\left(\sum_{i=1}^{m+r} d \mu_{i} \wedge d \theta_{i}\right)(X, Y)=0$.

If $X, Y \in T_{x} D \cap T_{x} S$ then $\omega_{S}(X, Y)=0$ because $D$ is co-Lagrangian, and $\left(\sum_{i=1}^{m+r} d \mu_{i} \wedge\right.$ $\left.d \theta_{i}\right)(X, Y)=0$ because $d \theta_{i}(X)=d \theta_{i}(Y)=0$ by construction.

If $X=\frac{\partial}{\partial \theta_{j}} \in T_{x} N$ and $Y \in T_{x} D \cup T_{x} S$ then by construction we also have

$$
\omega_{S}(X, Y)=\omega\left(\frac{\partial}{\partial \theta_{j}}, Y\right)=-d \mu_{j}(Y)=d \mu_{j} \wedge d \theta_{j}(X, Y)=\left(\sum_{i=1}^{m+r} d \mu_{i} \wedge d \theta_{i}\right)(X, Y)
$$

Since any vector pair $(X, Y) \in\left(T_{x} D \cap T_{x} S\right)^{2}$ can be decomposed into a linear combination of pairs of the above types, Formula (5.27) is proved.

Remark 5.19. The above theorem is the analog in the Dirac setting of the action-angle variables theorem for Hamiltonian systems on symplectic or Poisson manifolds which are integrable à la Liouville. In the symplectic case, the fibers of a regular Lagrangian fibration with compact fibers are automatically tori, but this fact is no longer true in the Dirac case: due to the degeneracy of the presymplectic forms on characteristic leaves, one can have non-torus Lagrangian fibrations with compact fibers on Dirac manifolds. So on a Dirac manifold we need not only a Lagrangian fibration, but also an integrable Hamiltonian system, in order to get action-angle variables.

5.6. Partial action-angle variables on Dirac manifolds. For non-commutatively integrable Hamiltonian systems on symplectic or Poisson manifolds, there are not enough action-angles variables to form a complete coordinate system, but one can complete these variables by some additional coordinates to form canonical coordinate systems (see [54, 53, 40] and previous sections of the present paper). The same is also true in the Dirac setting, when the Liouville tori are isotropic but not Lagrangian:

Theorem 5.20 (Partial action-angle variables). Let $N$ be a Liouville torus in an integrable Hamiltonian system $\left(X_{1}, \ldots, X_{p}, F_{1}, \ldots, F_{q}\right)$ on a regular Dirac manifold $(M, \mathcal{D})$ of bi-corank $(r, s)$, such that the distribution $T N \cap \mathcal{D}$ is regular of rank $d(0 \leq d \leq r)$ in a small tubular neighborhood $\mathcal{U}(N)$ of $N$ fibrated by Liouville tori. Then there is a coordinate system

$$
\left(\theta_{1}(\bmod 1), \ldots, \theta_{p}(\bmod 1), z_{1}, \ldots, z_{q}\right)
$$

on

$$
\mathcal{U}(N) \cong \mathbb{T}^{p} \times B^{q}
$$

and action functions

$$
\mu_{1}=z_{1}, \ldots, \mu_{p-d}=z_{p-d}, \mu_{p-d+1}, \ldots, \mu_{p}
$$


on $\mathcal{U}(N)$, such that the functions $\mu_{p-q+1}, \ldots, \mu_{p}$ depend only on the coordinates

$$
z_{1}, \ldots, z_{p-d}, z_{q-s+1}, \ldots, z_{q}
$$

the characteristic leaves of $\mathcal{D}$ in $\mathcal{U}(N)$ are

$$
S_{c_{1}, \ldots, c_{s}}=\left\{z_{q-s+1}=c_{1}, \ldots, z_{q}=c_{s}\right\},
$$

and the presymplectic form $\omega_{S}$ on each leaf $S=S_{c_{1}, \ldots, c_{s}}$ is of the form

$$
\omega_{S}=\left.\left(\sum_{i=1}^{p} d \mu_{i} \wedge d \theta_{i}\right)\right|_{S}+\left.\sum_{p-d<i<j \leq q-s-r+d} f_{i j} d z_{i} \wedge d z_{j}\right|_{S} .
$$

Proof. The proof is similar to the proof of Theorem 5.18. We can assume that TN is spanned by $\frac{\partial}{\partial \theta_{1}}, \ldots, \frac{\partial}{\partial \theta_{p-d}}$ and $T N \cap \mathcal{D}$. Then the action functions $\mu_{1}, \ldots, \mu_{p-d}$ are independent on the characteristic leaves, while the remaining action functions $\mu_{p-d+1}, \ldots, \mu_{p}$ are functionally dependent of $\mu_{1}, \ldots, \mu_{p-d}$ on each characteristic leaf, i.e., we can write $\mu_{p-d+1}, \ldots, \mu_{p}$ as functions of $\mu_{1}, \ldots, \mu_{p-d}, z_{q-s+1}, \ldots, z_{q}$, where the variables $z_{q-s+1}, \ldots, z_{q}$ are Casimir functions, and we can put $z_{1}=\mu_{1}, \ldots, z_{p-q}=\mu_{p-d}$.

Take a section $D$ (of dimension $q$ ) of the fibration by Liouville tori in $\mathcal{U}(N)$, with the following property: the image of $D$ by the projection proj: $\mathcal{U}(N) \rightarrow \mathcal{U}(N) / \mathcal{K}$, where $\mathcal{U}(N) / \mathcal{K}$ denotes the Poisson manifold which is the quotient of $\mathcal{U}(N)$ by the regular kernel foliation, is coisotropic of codimension $p-d$, and moreover the intersection of the kernel foliation with $\mathcal{D}$ is a regular foliation of dimension $r-d$ on $D$. We can choose the angle variables $\theta_{1}, \ldots, \theta_{p}$ so that they vanish on $D$.

The closed 1-forms $d \theta_{i}$ do not annul the kernel $T M \cap \mathcal{D}$ in general. But they do annul $T_{x} D \cap \mathcal{D}_{x}$ for any point $x \in D$ by construction. So for each $x \in D$, there are $p-d$ linear combinations $\sum_{j=1}^{p} c_{i j} d \theta_{j}(i=1, \ldots, p-d)$ which are linearly independent and which annul the kernel $T_{x} M \cap \mathcal{D}_{x}$. Hence there exist vectors

$$
Y_{1}(x), \ldots, Y_{p-d}(x) \in T_{x} M
$$

such that

$$
\left(Y_{i}(x), \sum_{j=1}^{p} c_{i j} d \theta_{j}(x)\right) \in \mathcal{D}_{x} \forall i=1, \ldots, p-d,
$$

and these vectors $Y_{1}(x), \ldots, Y_{p-d}(x)$ are linearly independent modulo the kernel $K_{x}=$ $\mathcal{D}_{x} \cap T_{x} M$ (i.e. no non-trivial linear combination of these vectors lies in $K_{x}$ ).

By the coisotropy property of $D$ (or more precisely, of the projection of $D$ in $\mathcal{U}(N) / \mathcal{K})$, we can choose $Y_{1}(x), \ldots, Y_{p-d}(x)$ so that they belong to $T_{x} D$. One verifies directly that the distribution $\mathcal{Y}$ on $D$ given by

$$
\mathcal{Y}_{x}=\operatorname{Span}_{\mathbb{R}}\left(Y_{1}(x), \ldots, Y_{p-d}(x)\right) \oplus\left(T_{x} D \cap \mathcal{D}_{x}\right)
$$

is an integrable regular distribution of dimension $p+r-2 d$.

Choose the $q-p+2 d-r$ coordinates $z_{p-d+1}, \ldots, z_{q-s-r+d}$ in such a way that they are constant on the Liouville tori, and also invariant with respect to the distribution $\mathcal{Y}$. Choose $r-d$ additional coordinates $z_{q-s-r+d+1}, \ldots, z_{q-s}$ such that they are also constant on the Liouville tori, and such that their differentials when restricted to the 
$(r-d)$-dimensional space $T_{x} D \cap \mathcal{D}_{x}$ form a basis of the dual space of that space for any point $x \in D$.

Finally, one verifies that $\omega_{S}-\left.\left(\sum_{i=1}^{p} d \mu_{i} \wedge d \theta_{i}\right)\right|_{S}$ can be expressed as

$$
\left.\sum_{p-d<i<j \leq q-s} f_{i j} d z_{i} \wedge d z_{j}\right|_{S}
$$

in a way similar to the end of the proof of Theorem 5.18

\section{TORUS ACTIONS AND ACTION-ANGLE VARIABLES NEAR SINGULARITIES}

In mechanics, by canonical coordinates many people often mean polar coordinates which are action-angle variables. For example, for the harmonic oscillator with the Hamiltonian $H=\left(x^{2}+y^{2}\right) / 2$ in canonical coordinates $(x, y)$, the action-angle variables $(h, \theta)$ where $h=H$ and $\theta=\arctan (x / y)$ are also often used as canonical coordinates.

In order to normalize a system near a singularity and find polar action-angle variables, we can follow the same approach which was used for action-angle variables near Liouville tori, and which consists of 3 steps:

- Intrinsic associated torus actions. Show the existence of a natural torus action (real or complex, formal or analytical or smooth) which is intrinsically associated to the system near a singularity. Here the word "singularity" may mean either a singular point of a vector field $X$ (where $X$ vanishes), or a singular orbit of the $\mathbb{R}^{p}$-action generated by the vector fields $X_{1}, \ldots, X_{p}$ of an integrable system $\left(X_{1}, \ldots, X_{p}, F_{1}, \ldots, F_{q}\right)$, or a singular level set of the system.

- Fundamental conservation property. Show that the associated torus action is a double commutant in the sense that "anything" which is preserved by the system will also be preserved by the torus action.

- Simultaneous normalization/linearization. Once we know that the associated torus action preserves an underlying geometric structure (e.g., a symplectic form), and we can linearize/normalize this geometric structure in an equivariant way with respect to the torus action (e.g., equivariant version of Darboux's theorem), then we get a normal form, which may contain some polar actionangle variables for the system. Even when there are no action variables, the normal form can still be very interesting.

Below let us show how this approach works in various situations.

6.1. Poincaré-Birkhoff normal forms and torus actions. Consider a formal or analytic vector field $X$ on $\mathbb{K}^{m}$, where $\mathbb{K}$ is $\mathbb{R}$ or $\mathbb{C}$, which vanishes at the origin $O$ of $\mathbb{K}^{m}$, and consider its Taylor expansion in some (formal or analytic) local coordinate system:

$$
X=X^{(1)}+X^{(2)}+\ldots
$$


The semi-simple part $X^{s s}$ of the linear part $X^{(1)}$ of $X$ is diagonalizable over complex numbers, so we can write

$$
X^{s s}=\sum_{i=1}^{m} \gamma_{i} z_{i} \frac{\partial}{\partial z_{i}}
$$

in some complex coordinate system $\left(z_{1}, \ldots, z_{m}\right)$. A nonlinear monomial vector field $z_{1}^{k_{1}} \ldots z_{m}^{k_{m}} \frac{\partial}{\partial z_{j}}$ is called resonant if

$$
\left[X^{s s}, z_{1}^{k_{1}} \ldots z_{m}^{k_{m}} \frac{\partial}{\partial z_{j}}\right]=0
$$

or equivalently, the multi-index tuple $\left(k_{1}, \ldots, k_{m}, i\right)$ satisfies the resonant relation

$$
\langle\gamma, k\rangle-\gamma_{i}:=\sum_{i=1}^{m} \gamma_{i} k_{i}-\gamma_{j}=0
$$

The classical formal normalization theorems of Poincaré, Birkhoff, Dulac, Gustavson and other authors say that there exists a coordinate system in which $X$ commutes with its simisimple linear part:

$$
\left[X, X^{s s}\right]=0
$$

or equivalently, all the non-zero nonlinear terms in the Taylor expansion of $X$ are resonant. The classical proofs of these theorems are based on the method of iterative normalization (elimination of non-resonant terms one by one). When $X$ is Hamiltonian on a symplectic space, then this normalization can be done symplectically, i.e. using canonical systems of coordinates and canonical transformations.

In particular, if $X=X_{H}$ is a Hamiltonian vector field on a symplectic space, with the Hamiltonian function $H=H^{(2)}+$ h.o.t. having its quadratic part

$$
H^{(2)}=\sum_{1}^{n} \gamma_{i} \frac{x_{i}^{2}+y_{i}^{2}}{2}
$$

nonresonant in a canonical coordinate system $\left(x_{i}, y_{i}\right)$ (which means that the numbers $\gamma_{1}, \ldots, \gamma_{n}$ are incommensurable), then all the resonant terms must be functions of the action variables $p_{i}=\frac{x_{i}^{2}+y_{i}^{2}}{2}$, and the Birkhoff normal form has the form

$$
H=h\left(\frac{x_{1}^{2}+y_{1}^{2}}{2}, \ldots, \frac{x_{n}^{2}+y_{n}^{2}}{2}\right),
$$

i.e., after a formal normalization, in the polar action-angle variables $\left(p_{1}, \theta_{1}, \ldots, p_{n}, \theta_{n}\right)$ with $p_{i}=\frac{x_{i}^{2}+y_{i}^{2}}{2}$ and $\theta_{i}=\arctan \left(x_{i} / y_{i}\right)$, the non-resonant Hamiltonian function $H$ depends only on the action variables $\left(p_{1}, \ldots, p_{n}\right)$ and is formally integrable. 
The minimal number $d$ such that the semisimple linear part $X^{s s}$ of $X$ can be written in the form

$$
X^{s s}=\sum_{i=1}^{d} \lambda_{i} Z_{i},
$$

where

$$
Z_{i}=\sum_{j=1}^{m} a_{i j} z_{j} \frac{\partial}{\partial z_{j}}
$$

are diagonal vector fields with integer coefficients $a_{i j} \in \mathbb{Z}$, is called the (complex formal) toric degree of $X$ at $O$. The minimality of $d$ is equivalent to the incommensurability of the numbers $\lambda_{1}, \ldots, \lambda_{d}$ in Equation (6.8).

In [72, 69] we showed the following results, under the above notations:

i) $X$ admits a natural associated system-preserving intrinsic torus $\mathbb{T}^{d}$-action, where $d$ is the (complex formal) toric degree of the system. This torus action is only formal in general, even when the system is analytic. In the case of Hamiltonian systems on symplectic manifolds, this torus action is also Hamiltonian.

ii) The normalization of $X$ à la Poincaré-Birkhoff is equivalent to the linearization of its associated torus action: the system is in its normal form if and only if this torus action is linear. Instead of step-by-step normalization for $x$, one can linearize this torus action using the averaging method with respect to compact group actions.

iii) $X$ admits a local analytic normalization if and only if its associated torus action is analytic (and not only formal).

iv) If the system is analytically integrable (i.e., it can be included in a integrable system $\left(X_{1}, \ldots, X_{p}, F_{1}, \ldots, F_{q}\right)$ whose components are all analytic) then its associated torus action is analytic. As a consequence, any analytic integrable system admits a local analytic Poincaré-Birkhoff normalization at any singular point.

This last result doesn't require any additional condition on (non)resonance, and is a significant improvement over previous results obtained by other authors for analytic integrable Hamiltonian systems: Rüssmann [57] (1964, the non-degenerate case with 2 degrees of freedom) Vey [61] (1978, the non-degenerate case), Ito [30] (1989, the nonresonant case), Ito 1992 [31] and Kappeler-Kodama-Némethi 1998 [34] (the cases with a simple resonance). For integrable Hamiltonian systems there are related results by Vey [62] and Stolotvitch [59, 60], among others, about the existence of local analytic normalization.

Similarly to the case of Liouville tori of integrable systems, the associated torus action of a formal or analytic vector field $X$ near a singular point also has the fundamental conservation property, even without any integrability condition. In particular, we have:

Theorem 6.1. Let $\mathcal{G}$ be an arbitrary formal tensor field which is invariant with respect to a formal vector field $X$ on $\mathbb{C}^{m}$ which vanishes at the origin $O$. Then the formal torus $\mathbb{T}^{d}$-action on $\mathbb{C}^{m}$ associated to $X$ (where $d$ is the toric degree of $X$ ) also preserves $\mathcal{G}$.

Proof. The proof is just a simple exercise in linear algebra and representation theory. We can assume that $X$ is in normal form: $\left[X, X^{s s}\right]=0$, where $X^{s s}$ is the semisimple 
linear part of $X$, and is already diagonalized in a coordinate system $\left(x_{i}\right)$. Then the monomial tensor fields in the coordinates $\left(x_{i}\right)$ are eigenvectors with respect to the Lie derivative operator $\mathcal{L}_{X^{s s}}$, i.e. if $\mathcal{H}$ is a monomial tensor field then $\mathcal{L}_{X^{s s}} \mathcal{H}=\lambda_{\mathcal{H}} \mathcal{H}$ for some $\lambda_{\mathcal{H}}$ which is a linear combination of the eigenvalues of $X$ with integral coefficients.

Assume that $\mathcal{L}_{X^{s s}} \mathcal{G} \neq 0$ for some tensor field, we will show that $\mathcal{L}_{X} \mathcal{G} \neq 0$. Let $\mathcal{H}$ be the monomial term with a non-zero coefficient $c_{\mathcal{H}}$ in $\mathcal{G}$ of lowest lexicographic order with respect to the Jordan decomposition $X^{(1)}=X^{s s}+X^{n i l}$, such that $\lambda_{\mathcal{H}} \neq 0$, where $X^{(1)}$ denotes the linear part of $X$ in the coordinate system $\left(x_{i}\right)$ and $X^{n i l}$ denotes the nilpotent part of $X^{(1)}$. It means that $\mathcal{H}$ has lowest degree in $\mathcal{G}$, and if $\mathcal{H}^{\prime}$ is another monomial tensor field such that $\left(L_{n}\right)^{k} \mathcal{H}^{\prime}=\mathcal{H}$ for some $k>0$, where $L_{n}$ denotes the nilpotent linear operator $\mathcal{A} \mapsto \mathcal{L}_{X^{\text {nil }}} \mathcal{A}$, then $\mathcal{H}^{\prime}$ does not appear in the expression of $\mathcal{G}$, i.e. its coefficient in $\mathcal{G}$ is zero. Due to the nilpotence of $\mathcal{L}_{X}-\mathcal{L}_{X^{s s}}$, the fact that $\mathcal{L}_{X}$ commutes with $\mathcal{L}_{X s s}$, and the choice of $\mathcal{H}$, we have that the coefficient of $\mathcal{H}$ in $\mathcal{L}_{X} \mathcal{G}$ is exactly equal to the coefficient of $\mathcal{H}$ in $\mathcal{L}_{X^{s s}} \mathcal{G}$, which is equal to $\lambda_{\mathcal{H}} \neq 0$.

Thus, in order for $\mathcal{L}_{X} \mathcal{G}$ to vanish, all monomial terms in $\mathcal{G}$ must be resonant with respect to $\mathcal{L}_{X s s}$, i.e., we must have $\mathcal{L}_{X s s} \mathcal{G}=0$.

Write $X^{s s}=\sum_{i=1}^{d} \lambda_{i} Z_{i}$, where $d$ is the toric degree of $X, Z_{i}=\sum a_{i j} x_{j} \partial x_{j}$ are diagonal linear vector fields with integral coefficients, and $\lambda_{1}, \ldots, \lambda_{d}$ are incommensurable numbers. The vector fields $\sqrt{-1} Z_{1}, \ldots, \sqrt{-1} Z_{d}$ are the generators of the associated torus action of $X$ (see [69, 72] ), and for each monomial tensor field $\mathcal{H}$ we have

$$
\mathcal{L}_{X^{s s}} \mathcal{H}=\sum_{i} \lambda_{i} \mathcal{L}_{Z_{i}} \mathcal{H}=\sum_{i} c_{i} m_{i} \mathcal{H}
$$

where $m_{i} \in \mathbb{Z}$ is the eigenvalue of $\mathcal{H}$ with respect to $\mathcal{L}_{Z_{i}}$. Since the numbers $c_{i}$ are incommensurable, we have that $\sum_{i} c_{i} m_{i}=0$ if and only if $m_{i}=0$ for every $i$, i.e. $\mathcal{L}_{Z_{i}} \mathcal{H}=0$ for every $i$. Thus, from $\mathcal{L}_{X^{s s}} \mathcal{G}=0$ we get $\mathcal{L}_{Z_{i}} \mathcal{G}=0$ for every $i$, which means that $\mathcal{G}$ is invariant with respect to the associated torus action.

In particular, if $X=X_{H}$ is a Hamiltonian vector field, and $\mathcal{G}$ is the symplectic form, then the associated torus action of $X$ automatically preserves $\mathcal{G}$, i.e. it is a Hamiltonian torus action. Because of this, Birkhoff normalization and Poincaré-Dulac normalization are essentially the same theory (see [73]).

The toric degree $d$ discussed above is also called complex toric degree, because even when $X$ is a real analytic vector field on $\mathbb{R}^{m}$, its associated torus $\mathbb{T}^{d}$-action acts on the complexified space $\mathbb{C}^{m}$ and not on the real space $\mathbb{R}^{m}$ in general. Only a subtorus of this complex torus action, say $\mathbb{T}^{d_{r}} \subset \mathbb{T}^{d}$ acts in the real space, and the (largest possible) number $d_{r}$ is called the real toric degree of $X$ at the origin $O$.

For example, if $X=X_{H}$ is a real integrable Hamiltonian vector field with a nonresonant singularity at $O$, then according to a classification by Williamson (see., e.g., [23, 51, 67]) and the local normalization theorem, the function $H$ can be written in some local analytic system of canonical coordinates as a real analytic function

$$
H=h\left(\mu_{1}, \ldots, \mu_{n}\right)
$$

of the components of $\mu_{i}$, which belong to 3 following types: 
- Elliptic $\mu_{j}=\frac{x_{j}^{2}+y_{j}^{2}}{2}$;

- Hyperbolic $\mu_{i}=x_{i} y_{i}$;

- Focus-Focus $\mu_{i}=x_{i} y_{i}+x_{i+1} y_{i+1}, \mu_{i+1}=x_{i} y_{i+1}-x_{i+1} y_{i}$.

The Williamson type of $X_{H}$ at $O$ is the triple $\left(k_{e}, k_{h}, k_{f}\right)$, where $k_{e}$ (resp., $k_{h}, k_{f}$ ) is the number of elliptic (resp., hyperbolic, focus-focus) components of $X$ at 0 . The complex toric degree of $X$ in this case is $n=k_{e}+k_{h}+2 k_{f}$, but its real toric degree is only $k_{e}+k_{f}$ : there is a local real analytic effective Hamiltonian torus $\mathbb{T}^{k_{e}+k_{f}}$ which preserves the system near $O$, and that number is maximal possible. If $\mathcal{G}$ is a real analytic tensor field (or more generally, a subbundle of a natural bundle) which is preserved by $X$, then its complexification is invariant under the torus $\mathbb{T}^{d}$-action in the complexified space, and hence $\mathcal{G}$ is also invariant under the associated action of $\mathbb{T}^{d_{r}}$ (where $d_{r}$ is the real toric degree) in the real space (which is a subaction of the associated torus action).

6.2. Torus actions near singular orbits and fibers. In [70, it was shown that, under a very mild so-called finite-type condition, near any $k$-dimensional compact orbit of a real analytic integrable system $\left(X_{1}, \ldots, X_{p}, F_{1}, \ldots, F_{q}\right)$ there is a $\mathbb{T}^{k}$-action which preserves the system and is transitive on the orbit. Together with the transverse toric degree $d$, which is the toric degree of the reduced system with respect to this $\mathbb{T}^{k}$-action we get an effective torus $\mathbb{T}^{k+d}$-action in a complexified neighborhood of the compact orbit, which plays the role of the associated torus action in this case, and Theorem 6.1 naturally extends to this associated torus action. As usual, the proof in the analytic case is simpler than in the smooth case, because we can make use of the natural filtration by the degree of the monomial terms.

For smooth but non-analytic integrable system, it does not make sense to talk about complexification, the torus action acts only in the real space (and so its dimension is smaller than the complex toric degree in general), the problem of normalization is harder in general, and one needs some nondegeneracy conditions (not just the finitetype condition which is too weak). Torus actions and partial action-angle variables for smooth integrable systems near singularities have been obtained by many authors, most notably Eliasson [23], Dufour-Molino [20] and Banyaga-Molino [5] (polar action-angle coordinates for elliptic singularities, on symplectic and contact manifolds), MirandaZung [52] (torus action and linearization near a nondegenerate compact orbit), and in our papers [67, 73]. Using topological arguments, it was shown in [67] (see also [73]) that, under some nondegeneracy conditions (which may be weakened) near a singular fiber of rank $k$ of a proper integrable Hamiltonian system on a symplectic manifolds there is a system-preserving Hamiltonian $\mathbb{T}^{k}$ action, which give rise to partial actionangle variables $\left(\mu_{i}, \theta_{i}\right)$ : the symplectic form $\omega$ has the form

$$
\omega=\sum_{i=1}^{k} d \mu_{i} \wedge d \theta_{i}+\beta,
$$

where $\beta$ does not depend on the variables $\left(\mu_{i}, \theta_{i}\right)$. 
Naturally, results of Section 2 about the fundamental conservation property extend to these smooth torus actions near singular orbits and fibers of proper integrable systems, because these torus actions are parts of Liouville torus actions outside of the singularities.

\section{SOME FINAL REMARKS}

In this paper, we studied only finite-dimensional classical dynamical systems generated by vector fields, but the same idea about associated torus actions playing the role of double commutants should also work in many other contexts. Let us mention here a few.

- Systems on Nambu manifolds. See [21] for an introduction to Nambu structures, and [74] for a more precise relationship between Nambu structures and singular foliations.

Let $\left(X_{1}, \ldots, X_{p}, F_{1}, \ldots, F_{q}\right)$ be an integrable system on a manifold $M$ and $\mathcal{D}$ be a smooth distribution on $M$ such that the vector fields $X_{i}$ are tangent to $\mathcal{D}$ and preserve $\mathcal{D}$. Then it follows easily from the fundamental conservation property that any Liouville torus $N$ of the system admits a tubular neighborhood $\mathcal{U}(N)$ which can be decomposed as

$$
\mathcal{U}(N) \cong \mathbb{T}^{p} \times B^{q},
$$

with $\mathcal{D}=T \mathbb{T}^{p} \oplus \mathcal{D}_{1}$, where $\mathcal{D}_{1}$ is a smooth distribution on $B^{q}$.

In the case when $\mathcal{D}$ is the associated distribution of an (almost) Nambu structure $\Lambda$ and $\mathcal{L}_{X_{i}} \Lambda=0$, then from the above splitting result for $\mathcal{D}$ we obtain the following splitting formula for $\Lambda$ :

$$
\Lambda=\frac{\partial}{\partial \theta_{1}} \wedge \ldots \wedge \frac{\partial}{\partial \theta_{p}} \wedge \Pi,
$$

where $\Pi$ is an (almost) Nambu structure on $B^{q}$, and $\theta_{1}, \ldots, \theta_{p}$ are action variables.

- Torus actions in normalizations of diffeomorphims and in the singular perturbation method (renormalization). Torus actions appear naturally not only in dynamical systems generated by vector fields, but also in diffeomorphisms, renormalization method, and so on, see, e.g., [55, 14. It is natural to expect that these natural torus actions also possess the fundamental conservation property.

- Infinite-dimensional integrable systems. If we have an integrable Hamiltonian system on an infinite dimensional symplectic manifold modeled on a separable Hilbert space, for example the periodic KdV or the integrable non-linear Schrödinger equation, then one observes the following phenomena (see, e.g., [36, 35, 39]):

i) The regular and singular elliptic level sets are compact tori homeomorphic (with the subspace topology) to $\mathbb{T}^{\mathbb{N}}$ with product topology or to a finite-dimensional torus. The tori $\mathcal{T}_{c}=\left\{z=\left.\left(z_{n}\right) \in \ell_{2}\left|z_{i} \in \mathbb{C},\right| z_{i}\right|^{2}=c_{i}\right\}$, where $c=\left(c_{n}\right)$ is a sequence of nonnegative real numbers whose sum converges, which appear in infinite-dimensional harmonic oscillators, are typical examples of such compact infinite-dimensional tori. 
(It is a simple exercise in elementary topology to show that the subspace topology of each such torus is the same as the product topology).

ii) There is an analytic infinite-dimensional Hamiltonian torus action near each Liouville or elliptic torus, which preserves the system and which gives rise to an infinitedimensional normal form à la Birkhoff, with an infinite number of action and angle variables.

- Quantum and semi-classical integrable systems. In a quantum mechanical system, angle variables are phases, action variables correspond to quantum numbers, and associated torus actions are periodic unitary transformations which preserve the system. (See, e.g., [43] for the example of quantum harmonic oscillator). The fundamental conservation property here means that any quantum operator which commutes with the system will also commute with the phase shift operators. In the semi-classical case, one has semi-classical Birkhoff normal forms (see, e.g., 64]), and there should exist associated torus actions, which preserve everything which is preserved by the semi-classical system, and whose linearization corresponds to the semi-classical normalization of the system.

\section{ACKNOWLEDGEMENT}

This paper is based on an unpublished preprint "Action-Angle variables on Dirac manifolds" arXiv:1204.3865) written by the author in 2012, which contains the central idea of the paper, namely action-angle normal forms can be easily obtained from the fundamental conservation property of the associated torus actions: everything which is preserved by the system is also preserved by the associated torus actions.

The results of this paper had been exposed in several talks at seminars and conferences, including the conference "Geometry and Mechanics" at Institut Henri Poincaré, Paris, 22-24/Nov/2014 in honour of C.-M. Marle, and the "Workshop on Poisson Geometry and Mathematical Physics" at Chern Institute, Tianjin, 04-08/Jan/2016. I would like to thank many colleagues for their interest in this work and for various discussions, and especially to Tudor Ratiu for encouraging me to write up this paper.

I finally found time to work out various details and to write up this paper in the first half of 2017, thanks to a stay at the School of Mathematical Sciences, Shanghai Jiao Tong University as a visiting professor. I would like to thank Shanghai Jiao Tong University, the colleagues at the School of Mathematics of this university, and especially Tudor Ratiu, Jianshu Li, and Jie Hu for the invitation, hospitality and excellent working conditions.

I would like also to thank the referees for many remarks which helped me to improve the presentation of the paper.

\section{COMPlianCE With ETHicAl STANDARdS}

This research did not involve any animals or human participants.

As far as I know, there is no conflict of interest; the main results presented in this paper are original and have not been submitted for publication in any other journal. 


\section{REFERENCES}

[1] R. Abraham, J.E. Marsden, Foundation of Mechanics, Second edition, revised and enlarged. With the assistance of Tudor Ratiu and Richard Cushman. Benjamin/Cummings Publishing Co., 1978.

[2] V. I. Arnold, Arnold's problems, Springer, 2004.

[3] V.I. Arnold, Mathematical Methods of Clasical Mechanics, 2nd edition, Chapter 10, Springer, 1989.

[4] V.I. Arnold, V.V. Kozlov, A.I. Neishtadt ed., Mathematical aspects of classical and celestial mechanics, vol.III of Dynamical Systems in the Ency- clopædia of Mathematical Sciences, Springer 1987

[5] A. Banyaga, P. Molino, Géométrie des formes de contact complètement intégrables de type toriques, Séminaire Gaston Darboux de Géométrie et Topologie Différentielle, 1991-1992 (Montpellier), 1-25.

[6] S. Bergia, L. Navarro, On the early history of Einstein's quantization rule of 1917, Archives internationales d'histoire des sciences, Vol. 50 (2000), No. 145, 321-373.

[7] G.D. Birkhoff, Dynamical Systems, 2nd ed., AMS Colloq. Publ., No. 9, Providence, 1927.

[8] O.I. Bogoyavlenskij, Extended integrability and bi-Hamiltonian systems, Comm. Math. Phys. 196 (1998), no. 1, 19-51.

[9] A. Bolsinov, J. Morales-Ruiz, N.T. Zung, Geometry and Dynamics of Integrable Systems, Advanced Courses in Mathematics - CRM Barcelona, Birkhäuser, 2016.

[10] C.P. Boyer, Completely Integrable Contact Hamiltonian Systems and Toric Contact Structures on $S^{2} \times S^{3}$, SIGMA 7 (2011), 058, 22 pages.

[11] H.W. Broer, M. B. Sevryuk, Chapter 6 - KAM theory: quasi-periodicity of dynamical systems, Hanbook of Dynamical Systems, Vol. 3 (2010), 249-344.

[12] J. M. Burgers. Die adiabatis hen Invarianten bedingt periodis her Systeme. Annalen der Physik, 357 (1917), No. 2, 195-202.

[13] H. Bursztyn, A brief introduction to Dirac manifolds, Chapter 1 of: Geometric and Topological Methods for Quantum Field Theory, Proceedings of the 2009 Villa de Leyva Summer School, Cambridge, 2013.

[14] H. Chiba, Extension and Unification of Singular Perturbation Methods for ODEs Based on the Renormalization Group Method, SIAM J. Appl. Dyn. Syst., 8 (2009), No. 3, 1066-1115.

[15] T. Courant, Dirac manifolds, Trans. Amer. Math. Soc. no. 319 (1990), 631-661.

[16] T. Courant, A. Weinstein, Beyond Poisson structures, Séminaire sudrhodanien de géométrie VIII. Travaux en Cours 27, Hermann, Paris (1988), 39-49.

[17] P. Dazord and T. Delzant, Le problème général des variables actions-angles, J. Differential Geom. 26 (1987), no. 2, 223-251.

[18] I. Ya. Dorfman, Dirac structures of integrable evolution equations, Phys. Lett. A 125 (1987), no. 5, 240-246.

[19] H.S. Dumas, The KAM Story, World Scientific, 2014.

[20] J.-P. Dufour, P. Molino, Compactification d'action de $\mathbb{R}^{n}$ et variables action-angle avec singularités, MSRI Publ., Vol. 20 (1990) (Séminaire Sud-Rhodanien de Géométrie, Berkeley, 1989, P. Dazord and A. Weinstein eds.), 151-167

[21] J.P. Dufour, N.T. Zung, Poisson structures and their normal forms, Progress in Mathematics, Vol. 242, 2005.

[22] J.J. Duistermaat, On global action-angle variables, Comm. Pure Appl. Math., 33 (1980), 687-706.

[23] H. Eliasson, Normal form of Hamiltonian systems with Poisson commuting integrals - elliptic case, Comment. Math. Helv. 65 (1990), 4-35.

[24] A. Einstein, Zum Quantensatz von Sommerfeld und Epstein, Verhandlungen der Deutschen Physikalischen Gesellschaft, 19 (1917), 82-92.

[25] F. Fassò, N. Sansonetto, Integrable almost-symplectic Hamiltonian systems, J. Math. Phys. 48 (2007), no. 9, 092902, 13 pp.

[26] J. Féjoz, On action-angle coordinates and on the Poincaré coordinates, Regular and Chaotic Dynamics, 18 (2013), No. 6, pp. 703-718.

[27] I. M. Gelfand, I. Ya. Dorfman, Hamiltonian operators and algebraic structures related to them, Funct. Anal. Appl. 13 (1979), 248-262. 
[28] V. Guillemin, S. Sternberg, Symplectic techniques in physics, Cambridge University Press, Cambridge, second edition, 1990.

[29] H. Hofer, E. Zehnder, Symplectic Invariants and Hamiltonian Dynamics, reprint of the 1994 edition, Modern Birkhäuser Classics. Birkhäuser Verlag, Basel, 2011.

[30] H. Ito, Convergence of Birkhoff normal forms for integrable systems, Comment. Math. Helv., 64 (1989), 412-461.

[31] H. Ito, Integrability of Hamiltonian systems and Birkhoff normal forms in the simple resonance case, Math. Ann., 292 (1992), No. 3, 411-444.

[32] B. Jovanovic, Noncommutative integrability and action-angle variables in contact geometry, $J$. Symplectic Geom. Volume 10 (2012), Number 4, 535-561.

[33] B. Jovanovic, V. Jovanovic, Contact Flows and Integrable Systems, J. Geom. Phys., 87 (2015), 217-232.

[34] T. Kappeler, Y. Kodama, A. Némethi, On the Birkhoff normal form of a completely integrable Hamiltonian system near a fixed point with resonance, Ann. Scuola Norm. Sup. Pisa Cl. Sci. (4), Vol. XXVI (1998), 623-661.

[35] T. Kappeler, P. Lohrmann, P. Topalov, N.T. Zung, Birkhoff coordinates for the focusing NLS equation, Comm. Math. Phys. 285 (2009), no. 3, 1087-1107.

[36] T. Kappeler, J. Pöschel, $K d V$ and $K A M$, Ergebnisse der Mathematik und ihrer Grenzgebiete, Volume 45, Springer, 2003.

[37] B. Khesin and S. Tabachnikov, Contact complete integrability, Regular and Chaotic Dynamics, 15 (2010), no. 4-5, 504-520.

[38] Y. Kosmann-Schwarzbach, Dirac pairs, J. Geom. Mech. 4 (2012), no.2, 165-180.

[39] S. Kuksin, G. Perelman, Vey theorem in infinite dimensions and its application to KdV, Discrete and Continuous Dynamical Systems - Series A, 27 (2010), No. 1, 1-24.

[40] C. Laurent-Gengoux, E. Miranda, P. Vanhaecke, Action-angle coordinates for integrable systems on Poisson manifolds, Int. Math. Res. Notices 8 (2011), 1839-1869.

[41] E. Lerman, Contact Toric Manifolds, J. of Symplectic Geometry 1 (2002), No. 4, 785-828

[42] T. Levi-Civita. Sugli invarianti adiabati, Atti del Congresso internazionale dei Fisici, 1927.

[43] H.R. Lewis, W.E. Lawrence, J.D. Harris, Quantum Action-Angle Variables for the Harmonic Oscillator, Physical Review Letters 77 (1997), No. 26, 5157-5159.

[44] C. Libermann, Legendre foliations on contact manifolds, Differential Geometry and its Applications, 1 (1991), Issue 1, 57-76.

[45] P. Libermann, C.M. Marle, Symplectic Geometry and Analytical Mechanics, Kluwer Academic Publishers, 1987.

[46] J. Liouville, Note sur l'intégration des équations differentielles de la dynamique, présentée au bureau des longitudes le 29 juin 1853, Journal de Mathématiques pures et appliquées 20 (1855), $137-138$

[47] L. Markus, K. Meyer, Generic Hamiltonian dynamical systems are neither integrable nor ergodic, Memoirs of the AMS, 144 (1974), Chapter 3.

[48] H. Mineur, Sur les systèmes mécaniques admettant n intégrales premières uniformes et l'extension a ces systèmes de la méthode de quantification de Sommerfeld, C. R. Acad. Sci. Paris 200 (1935), $1571-1573$.

[49] H. Mineur, Sur les systèmes mécaniques dans lesquels figurent des paramètres fonctions du temps. Etude des systèmes admettant $n$ intégrales premières uniformes en involution. Extension a ces systèmes des conditions de quantification de Bohr-Sommerfeld, Journal de l'Ecole Polytechnique III, 143 (1937), 173-191 and 237-270.

[50] N.V. Minh, N.T. Zung, Geometry of integrable dynamical systems on 2-dimensional surfaces, Acta Mathematica Vietnamica, 38 (2013), No. 1.

[51] E. Miranda, On Symplectic Linearization of Singular Lagrangian Foliations, Ph.D. thesis, 2003.

[52] E. Miranda, Nguyen Tien Zung, Equivariant normal form for nondegenerate singular orbits of integrable Hamiltonian systems, Ann. Sci. École Norm. Sup. (4) 37 (2004), no. 6, 819-839.

[53] A. S. Mishchenko, A. T. Fomenko, Generalized Liouville method of integration of Hamiltonian systems, Funct. Anal. Appl. 12 (1978), 113-121. 
[54] N. N. Nekhoroshev, Action-angle variables and their generalizations, Trans. Moskow Math. Soc. 26 (1972), 180-198.

[55] J. Raissy, Torus action in the normalization problem, Journal of Geometric Analysis, 20 (2010), $472-524$.

[56] T. Ratiu, N.T. Zung, Presymplectic convexity and (ir)rational polytopes, preprint arXiv:1705.11110 (2017).

[57] H. Rüssmann, Uber das Verhalten analytischer Hamiltonscher Differentia " lgleichungen in der Nähe einer Gleichgewichtslösung, Math. Ann., 154 (1964), 285-300.

[58] P. Ševera, A. Weinstein, Poisson geometry with a 3-form background, Prog. Theor. Phys. 144 (2002), 145-154.

[59] L. Stolovitch, Singular complete integrability, Publications IHES, 91 (2000), 134-210.

[60] L. Stolovitch. Normalisation holomorphe d'algèbres de type Cartan de champs de vecteurs holomorphes singuliers, Ann. of Math., 161 (2005), 589-612.

[61] J. Vey, Sur certaines systèmes dynamiques séparables, Amer. J. Math., 100 (1978), 591-614.

[62] J. Vey, Algèbres commutatives de champs de vecteurs isochores, Bull. Soc. Math. France, 107 (1979), 423-432.

[63] S. M. Webster, Quadrics and complete integrability in contact geometry, Commun. Pure Appl. Math. 56 (2003), Issue 7, 824-838.

[64] Vũ Ngoc San, Symplectic techniques for semiclassical completely integrable systems, in Topological Methods in the Theory of Integrable Systems, Editors A.V. Bolsinov, A.T. Fomenko and A.A. Oshemkov, Cambridge Scientific Publications (2006).

[65] A. Weinstein, Lectures on symplectic manifolds, Conference Board of the Mathematical Sciences, No. 29 (1977).

[66] A. Weinstein, The local structure of Poisson manifolds. J. Differential Geom. 18 (1983), no. 3, $523-557$.

[67] N.T. Zung, Symplectic topology of integrable Hamiltonian systems. I. Arnold-Liouville with singularities, Compositio Math. 101 (1996), no. 2, 179-215.

[68] N.T. Zung, Kolmogorov condition for integrable systems with focus-focus singularities, Phys. Lett. A 215 (1996), no. 1-2, 40-44.

[69] N. T. Zung, Convergence versus integrability in Poincaré-Dulac normal form, Math. Res. Lett. 9 (2002), no. 2-3, 217-228.

[70] N.T. Zung, Actions toriques et groupes d'automorphismes de singularités des systèmes dynamiques intégrables, Comptes Rendus Mathématiques 336 (2003), Issue 12, pages 1015-1020.

[71] N.T. Zung, Symplectic topology of integrable Hamiltonian systems II. Topological classification, Compositio Math., 138(2) (2003), 125-156.

[72] N.T. Zung, Convergence versus integrability in Birkhoff normal form, Ann. of Math. (2) 161 (2005), no. 1, 141-156.

[73] N.T. Zung, Torus actions and integrable systems, in Topological Methods in the Theory of Integrable Systems, Editors A.V. Bolsinov, A.T. Fomenko and A.A. Oshemkov, Cambridge Scientific Publications (2006), 289-328.

[74] N.T. Zung, T.H. Minh, Commuting foliations, Regular and Chaotic Dynamics 18 (2013), Issue 6, pp 608-622.

[75] N.T. Zung, N.T. Thien, Reduction and integrability of stochastic dynamical systems, Fundamentalnaya i Prikladnaya Matematika (Fundamental and Applied Mathematics), 20 (2015), Number $3,213-249$.

School of Mathematics, Shanghai Jiao Tong University (visiting professor), 800 Dongchuan Road, Minhang District, Shanghai, 200240 China

Institut de Mathématiques de Toulouse, UMR5219, Université Paul Sabatier, 118 route de Narbonne, 31062 Toulouse, France

E-mail address: tienzung@math.univ-toulouse.fr 